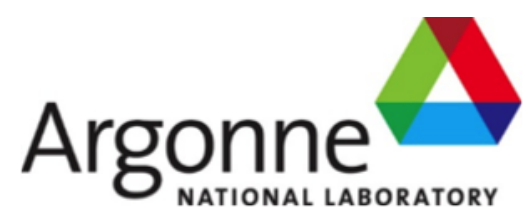

\title{
Identify the influence of microstructure on mesoscale creep and fatigue damage
}

Applied Materials Division 
About Argonne National Laboratory

Argonne is a U.S. Department of Energy laboratory managed by UChicago Argonne, LLC under contract DE-AC02-06CH11357. The Laboratory's main facility is outside Chicago, at 9700 South Cass Avenue, Argonne, Illinois 60439. For information about Argonne and its pioneering science and technology programs, see www.anl.gov.

\section{DOCUMENT AVAILABILITY}

Online Access: U.S. Department of Energy (DOE) reports produced after 1991 and a growing number of pre-1991 documents are available free at OSTI.GOV (http://www.osti.gov/), a service of the U.S. Dept. of Energy's Office of Scientific and Technical Information

Reports not in digital format may be purchased by the public from the

National Technical Information Service (NTIS):

U.S. Department of Commerce

National Technical Information Service

5301 Shawnee Rd

Alexandria, VA 22312

www.ntis.gov

Phone: (800) 553-NTIS (6847) or (703) 605-6000

Fax: (703) 605-6900

Email: orders@ntis.gov

Reports not in digital format are available to DOE and DOE contractors from the Office of Scientific and Technical Information (OSTI)

U.S. Department of Energy

Office of Scientific and Technical Information

P.O. Box 62

Oak Ridge, TN 37831-0062

www.osti.gov

Phone: (865) 576-8401

Fax: (865) 576-5728

Email: reports@osti.gov

\section{Disclaimer}

This report was prepared as an account of work sponsored by an agency of the United States Government. Neither the United States Government nor any agency thereof, nor UChicago Argonne, LLC, nor any of their employees or officers, makes any warranty, express or implied, or assumes any legal liability or responsibility for the accuracy, completeness, or usefulness of any information, apparatus, product, or process disclosed, or represents that its use would not infringe privately owned rights. Reference herein to any specific commercial product, process, or service by trade name, trademark, manufacturer, or otherwise, does not necessarily constitute or imply its endorsement, recommendation, or favoring by the United States Government or any agency thereof. The views and opinions of document authors expressed herein do not necessarily state or reflect those of the United States Government or any agency thereof, Argonne National Laboratory, or UChicago Argonne, LLC. 


\section{Identify the influence of microstructure on mesoscale creep and fatigue damage}

Applied Materials Division

Argonne National Laboratory

September 2020

Prepared by

A. Rovinelli, Argonne National Laboratory

M. C. Messner, Argonne National Laboratory 



\section{Abstract}

This report describes the development of a microstructural model that can quantify the uncertainty in the observed rupture life of Grade 91 steel. The model is microstructural, meaning it relates microstructural characteristics of the material to the resulting material response. As such, one of the uses of this model is to identify the key microstructural parameters controlling the development of damage in Grade 91 operating at elevated temperatures. The report describes two veins of work: improvements to the crystal plasticity model required to run the uncertainty quantification analysis and the results of that UQ analysis. For creep, the model identifies the grain boundary diffusivity as the critical parameter controlling the rupture life of the material. The report demonstrates that a reasonable microstructural distribution of grain boundary diffusivity can account for the observed macroscale variation in rupture life at fixed temperature and load. 



\section{Table of Contents}

Abstract i i

Table of Contents $\quad$ iii

List of Figures $\quad$ V

List of Tables vii

1 Introduction 1

2 Large Deformation Hill-Mandel Cell Conditions in MOOSE 5

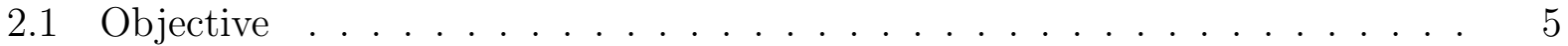

2.2 Enforcing cell average and Hill-Mandel conditions . . . . . . . . . . . . . . 7

2.2 .1 Previous work . . . . . . . . . . . . . . . . . 7

2.2 .2 New formulation . . . . . . . . . . . . . . 8

2.2.3 Problems with the basic formulation . . . . . . . . . . . . . . . 9

2.3 Total Lagrangian kernel . . . . . . . . . . . . . . . . . . . . . . . . . . . . . . . . . . . . . . . .

2.3.1 Residual equation . . . . . . . . . . . . . . . . . . . 11

2.3.2 Jacobian terms . . . . . . . . . . . . . . . . . . . . . . . . 11

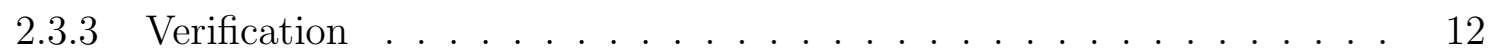

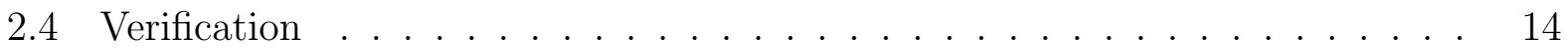

2.5 Identification of periodic boundaries . . . . . . . . . . . . . . 15

3 Large deformation cohesive zone model in MOOSE $\quad 19$

3.1 Implementation in MOOSE . . . . . . . . . . . . . . . . . . . . . . 21

3.1.1 Cohesive model residual . . . . . . . . . . . . . . . . . . . . 21

3.1.2 Cohesive model Jacobian . . . . . . . . . . . . . . . . . . . . . . . . . . . . . . . . . . 22

3.2 Validation . . . . . . . . . . . . . . . . . . 26

3.2.1 Objective traction rate validation . . . . . . . . . . . . . 26

3.2.2 Cohesive zone model Jacobian validation . . . . . . . . . . . . . . . 26

4 Constitutive models 33

4.1 The prior austenite grain model . . . . . . . . . . . . . . . . . . . 33

4.2 Grain boundary cavitation model . . . . . . . . . . . . . . . 34

5 Identification of microstructural parameters influencing creep rupture life 39

5.1 Sensitivity analysis . . . . . . . . . . . . . . . . . . . . . . . . . . . . . . . . . . 49

5.2 Identifying parameters distributions . . . . . . . . . . . . . . . 45

6 Conclusions and future work 49

Acknowledgments $\quad 51$

$\begin{array}{ll}\text { Bibliography } & 53\end{array}$ 



\section{List of Figures}

2.1 Illustration of the potential problems with box-sided representative cells. (a) Box-sided cell. (b) Equivalent true-periodic cell. (c) Periodic tiling of the box-sided cell with the artificially implied grain boundaries highlighted. (d) Periodic tiling of the true-periodic cell. The highlighted periodic grain boundaries are realistic. . . . . . . . . . . . . . . . . . . . .

2.2 Comparison between the updated and total Lagrangian formulations for a simple forming problem. (a) Deformed shape for the updated Lagrangian formulation. (b) Deformed shape for the total Lagrangian formulation. (c) Total dissipated energy (work) plotted as a function of bend angle for the two formulations. The two lines exactly overlap. . . . . . . . . . . . . . . .

2.3 (a) Simple truncated octahedron cell for the verification calculation. (b) Demonstration that the cell remains periodic after significant deformation.

2.4 Plots of the cell-average stress and deformation, demonstrating that the model obeys the imposed constraints. . . . . . . . . . . . . . . .

2.5 Render of a synthetic periodic microstructures with 100 grains, generated with Neper, colored by grain number.

2.6 Render of a synthetic periodic microstructures highlighting the identified periodic surfaces. Different colors are associated to different periodic surfaces.

3.1 Two dimensional schematic depicting the three surface, denoted by,+- and * symbols, used in the large deformation cohesive zone model along with the locations of the available integration points, e.g. blue squares. The + and - surfaces are the surfaces of two adjacent elements defining the cohesive interface. The $*$ surface represents the cohesive interface mid-plane. Notice that, the,+- and $*$ surfaces are coincident in the undeformed configuration but they are drawn as separated for illustration purposes. . . . . . . . . . . .

3.2 Rendering of the simulation without the CZM interface at different point in time. Axial stretching starts at time $=0$ and ends a time $=1$. The $90^{\circ}$ rigid body rotation around the $y$ axis starts at time $=1$ and ends a time $=2$. The highlighted surface shows where the traction are computed. . . . . . . .

3.3 Comparison of the First Piola-Kirchoff traction for the two simulations with and without cohesive zone linear elastic model. Loading conditions are described in Figure 3.2. . . . . . . . . . . . . . . . . . . . .

3.4 Comparison of the Cauchy traction for the two simulations with and without cohesive zone linear elastic model. Loading conditions are described in 3.2. .

3.5 Render of the patch test mesh used to check the correctness of the implemented analytic Jacobian. Cohesive interfaces have been inserted between each and every element. . . . . . . . . . . . . . . . . . . . .

4.1 Schematic representing the physical meaning of the grain boundary cavitation model state variables. The variable $a$ represents the average cavity half radius, and the variable $b$ represents the average cavity half spacing. . . . . . . . . . 
5.1 Experimentally observed distribution of Grade 91 creep rupture life at $600{ }^{\circ} \mathrm{C}$ for a nominal stress of $100 \mathrm{MPa}$. The black line represents simulation results

5.2 Schematic representing box-periodic boundary conditions for uniaxial creep loading. The colored block represents the RVE and each color represents a grain. $T_{Z}$ is the nominal traction and corresponds to a constant applied force. 41

5.3 Rendering of simulation results for uniaxial creep and a nominal stress of $100 \mathrm{MPa}$. . . . . . . . . . . . . . . . . . . . . . .

5.4 Sensitivity of different failure metrics with respect to the model parameters when performing a one-at-a-time sensitivity analysis. The failure metrics are: i) time to $1 \%$ interface strain (TT1), ii) time to $2 \%$ interface strain (TT2), and iii) time to $3 \%$ interface strain (TT3). Sensitivity is plotted in log space.

5.5 Computed time to failure, using the time to $1 \%$ interface strain metric, as function of the grain boundary diffusivity. . . . . . . . . . . . . .

5.6 The numerically computed grain boundary diffusivity distribution. Vertical line represent the calibrated $D_{G} B$ value. The blue line represents a smooth distribution calculated using the kernel density estimation. . . . . . . . . . .

5.7 Normalized grain boundary energy. The dashed line shows the variability of the energy for random grain boundaries. Data from $[31] \ldots \ldots \ldots$ 


\section{List of Tables}

2.1 Number of extra scalar degrees of freedom and corresponding cell average constraints required for different cell formulations. . . . . . . . . . . . . . . .

3.1 Comparison of number of nonlinear iteration required to achieve convergence when using: i) the finite difference calculated Jacobian (column FDJ), and ii) the analytic Jacobian $($ column AJ) . . . . . . . . . . . . . 31

4.1 Grain bulk material parameters. . . . . . . . . . . . . . . . 34

4.2 Grain boundary cavitation material parameters. . . . . . . . . . . . . 37 



\section{Introduction}

This report describes an effort at Argonne National Laboratory (ANL) to quantify the key microstructural characteristics linked to creep deformation and damage in Grade 91 steel. The work described here is part of a larger effort sponsored though the Nuclear Energy Advanced Modeling and Simulation (NEAMS) program at ANL, Idaho National Laboratory (INL), and Las Alamos National Laboratory (LANL) to address the critical need to better quantify uncertainty in the design of nuclear reactor structural components. This work feeds into the Grizzly simulation platform, developed at INL, which is a tool for structural analysis, including uncertainty quantification, for reactor components.

The current work fits into this broader approach by quantifying uncertainty in long term creep deformation and rupture in Grade 91. Grade 91 steel is a ferritic-martensitic alloy slated for future use in advanced, high temperature reactors because of its low cost, good thermal properties, and relatively good long-term creep strength [1,2]. The current design process for reactor structural components applies a factored, deterministic analysis to bound the expected distribution of component loading, geometric imperfections, and, crucially, material properties. However, our understanding of the variability in key material properties for Grade 91 and other structural materials is limited, particularly for long service lives, exposure to corrosive coolants and radiation damage, and for out-of-normal operating conditions. Current practice develops design material properties through direct testing, which necessarily limits the test durations and environmental conditions for which data is available. Empirical extrapolation helps extend this test database to longer component service lives, but a better approach will be required for future advanced reactors. In particular, better uncertainty quantification and the development of statistical design approaches for high temperature structural components could lead to more efficient, economical component designs by tailoring the design of the component to required component reliability, rather than simply applying a constant, generally quite conservative, design margin to a large spectrum of different components.

The work described here applies a physically-based model to help determine key microstructural characteristics leading to long-term creep deformation and failure in Grade 91. This physically-based approach provides a natural method for quantifying uncertainty in key material properties. If a model can relate microstructural features to material performance then the distribution of these microstructural features can be linked to the expected distribution of critical material properties. The key point is that quantifying these microstructural characteristics does not require long-term testing.

The current work builds on a deterministic crystal plasticity finite element method (CPFEM) model for creep deformation and rupture in Grade 91 [3, 4] developed through past work in the Advanced Reactor Technologies (ART) program. This past work developed the model to make deterministic predictions for long-term creep in Grade 91. This model has been transferred to the MOOSE framework and we use it here as a basis for a statistical, microstructural model aimed at quantifying uncertainty in long-term properties.

This work is synergistic with companion work at LANL and INL. All the NEAMS structural efforts will ultimately end up as material models and methods in the Grizzly framework. LANL is developing a similar microstructural model using a different numerical approach (the crystal plasticity fast Fourier transform, CPFFT, approach) and developing fast surrogate models to embed microstructurally-informed models in the Grizzly framework. The CPFFT 
approach is more numerically efficient and so better suited at addressing microstructural uncertainties, for example through large numbers of Monte Carlo simulations. However, the microstructural sensitivity in the model formulation developed here using CPFEM should be directly relevant to LANL's efforts as it concerns the identification of key microstructural parameters, which are independent of the numerical simulation technique. Moreever, there are benefits to maintaining two mesoscale simulation solvers, i.e. both CPFEM and CPFFT:

- CPFEM is better suited to model complex interfaces, including complicated grain boundary geometries.

- Having two solvers means new features in one solver can be validated against the second. The equivalance of the two simulation techniques for bulk polycrystal deformation is not in doubt [5], but in particular, integrating grain boundary cavitation models into CPFFT calculations requires the development of new numerical methods, which could be validated against the well-established CPFEM discretization scheme.

- The CPFEM method builds off standard finite element methods and so improvements to the CPFEM framework translate to generic FE improvements to Grizzly. Similarly, the ANL CPFEM model uses the existing, well-developed MOOSE finite element framework, for example for implementing the interface damage model.

A similar microstructural sensitivity study could be applied to deterministic microstructural creep-fatigue damage. Such a model is under development, currently through the ART program. However, an early decision was made to focus the joint INL/ANL/LANL work on creep rupture, at least for the first year of this new program on structural materials.

This report then describes two veins of work:

1. Improvements to the MOOSE/CPFEM framework specifically aimed at accomplishing the microstructural sensitivity study.

2. The results of the sensitivity study, including the identification of the key microstructural properties contributing to creep damage in Grade 91.

Chapter 2 describes the development of a method for applying generic cell-average boundary conditions to arbitrarily-shaped, periodic simulation cells obeying the Hill-Mandel conditions. This allows us to use simulation volumes generated through periodic Voronoi tessellation, avoiding "box-sided" simulation cells which introduce artificial grain boundary geometries. As described in Chapter 2, this required a fundamental restructuring of the large deformation solid mechanics kernel in MOOSE to use a total Lagrangian formulation. This work includes Section 2.5, which describes the implementation of a MOOSE MeshGenerator that automatically detects periodic boundaries in periodic Voronoi tessellations.

Chapter 3 describes the incorporation of a large deformation cohesive zone framework able to account for large area changes and rotations. As discussed in the chapter, the cohesive zone framework has been developed using a total Lagrangian formulation and includes the exact Jacobian. Chapter 4 is a quick overview of the constitutive models used to describe deformation in the prior austenite grains and the grain boundary cavitation model. This is not new work and the aim of this chapter is to provide the reader with enough information to understand the results of the sensitivity analysis. 
Chapter 5 describes the methodology used to model the experimental uncertainty in the creep-rupture life of Grade 91 steel. Section 5.1 identifies the key microstructural parameters through sensitivity analysis and relates those parameters to the physics embedded in the microstructural model. The sensitivity study shows that the controlling model parameter is the grain boundary diffusivity. Section 5.2 describes how the sensitivity analysis results are used to identify the model parameter distributions that explain the macroscale rupture data. Results of this section show that accounting for the uncertainty of the grain boundary diffusivity is sufficient to capture the experimental uncertainty of the creep-rupture life of Grade 91.

Finally, Chapter 6 summarizes the work and describes the remaining work required to account for additional sources of uncertainty, such as the second phase particle distribution at the prior austenite grain boundaries. 



\section{Large Deformation Hill-Mandel Cell Conditions in MOOSE}

\subsection{Objective}

Figure 2.1 shows two notionally-identical simulation cells, intended for use as periodic representative volumes in a microstructural simulation. Both were generated using the Neper [6] tool for producing representative microstructures using periodic Voronoi tessellation. Both microstructures have identical grain orientations and grain shapes within the cell. Both are periodic in the sense that both cells will tile space. The difference is Figure 2.1(a) is "box-sided" - the tessellation was truncated to make a cubic cell. Figure 2.1(b) is the fully periodic tessellation.

This chapter discusses changes to MOOSE required to run simulations based on cells like Figure 2.1(b). Figure 2.1 (c) and (d) illustrates the reason why. Box-sided cells introduce a set of grain boundaries on the cell faces with an unphysical geometry - an entire plane of aligned grain boundaries - whereas the true-periodic cells do not introduce similar sets of planar boundaries. These artificial boundaries are not important for simulations including only grain bulk deformation. However, they can be important for simulations with grain boundary physics, like the creep cavitation model. The entire set of boundaries could, for example, directly align with the applied stress, creating a weak plane in the simulated volume.

Running simulations with these types of cells, in the context of proper homogenization theory, requires two conditions:

1. The cell obeys the classical Hill-Mandel condition.

2. A mechanism for enforcing arbitrary cell-averaged conditions on the simulation. Ideally these conditions would include arbitrary combinations of stress and strain constraints in different directions.

This chapter describes a mechanism for enforcing both conditions in MOOSE in the context of large deformation kinematics.

The Hill-Mandel condition [7,8] provides the necessary condition for the admissibility of the homogenized stress and deformation fields:

$$
\langle\boldsymbol{\sigma}: \boldsymbol{D}\rangle=\langle\boldsymbol{\sigma}\rangle:\langle\boldsymbol{D}\rangle
$$

where $\boldsymbol{\sigma}$ is the Cauchy stress, $\boldsymbol{D}$ is the deformation rate, and

$$
\langle X\rangle=\frac{1}{V} \int_{V} X d V
$$

Typically, models enforce the Hill-Mandel constraint using boundary conditions on the simulation cell surface. There are many cell conditions that meet the Hill-Mandel condition, but the most common are:

1. Uniform traction $\mathrm{BC}: \sigma \cdot \mathbf{n}=\hat{\mathbf{t}}$

2. Uniform displacement $\mathrm{BC}: \mathbf{u}=\hat{\mathbf{u}}$

3. Periodic displacements: $\mathbf{u}_{+}-\mathbf{u}_{-}=\mathbf{0}$, where + and - indicate two pairs of periodic faces. 
(a)
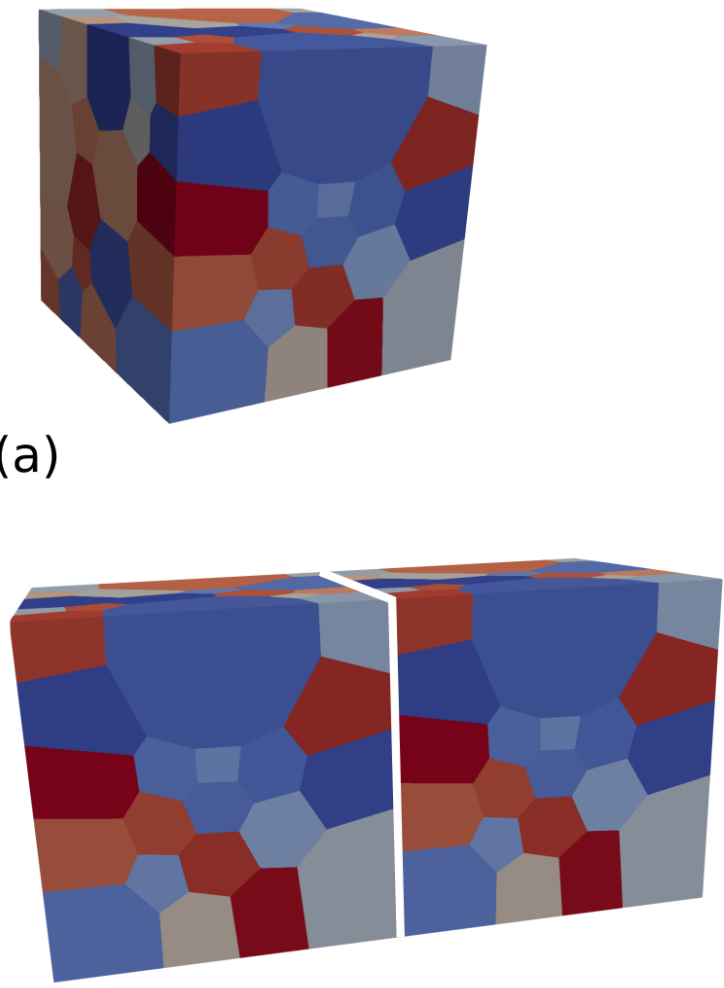

(c) (b)
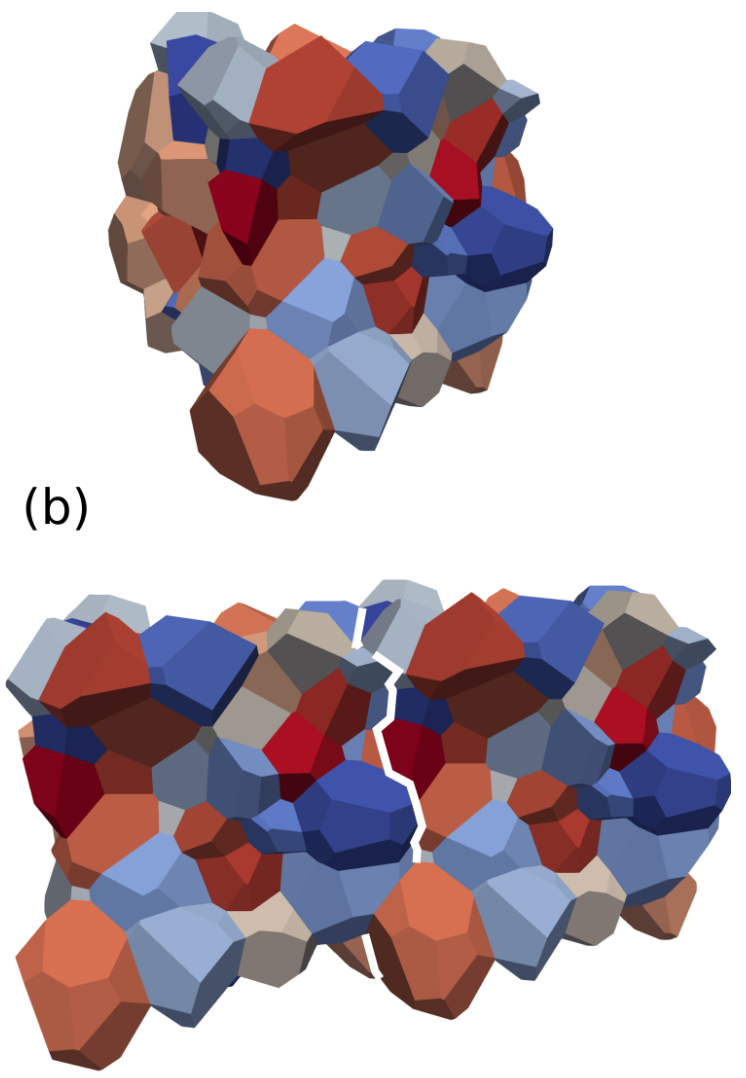

(d)

Figure 2.1: Illustration of the potential problems with box-sided representative cells. (a) Box-sided cell. (b) Equivalent true-periodic cell. (c) Periodic tiling of the box-sided cell with the artificially implied grain boundaries highlighted. (d) Periodic tiling of the trueperiodic cell. The highlighted periodic grain boundaries are realistic. 
Periodic boundary conditions are often the most efficient in determining effective properties from small representative volume elements. As such, this chapter seeks a solution that imposes periodic conditions together with imposed cell-average constraints.

In addition to these boundary conditions some types of uniform cell conditions also satisfy the constraint:

1. Uniform stress: the Ruess bound

2. Uniform deformation: the Voigt bound.

Superpositions of these conditions will also satisfy the Hill-Mandel condition.

For large deformations, cell-average constraints take the form:

$$
\begin{aligned}
& \hat{\sigma}_{i j}=\frac{1}{V} \int_{V} \sigma_{i j} d V \\
& \hat{F}_{i j}=\frac{1}{V} \int_{V} F_{i j} d V
\end{aligned}
$$

However, creep conditions impose a fixed dead load on the specimen, not a fixed Cauchy stress. So instead, this chapter focuses on stress constraints of the type

$$
\hat{P}_{i j}=\frac{1}{V} \int_{V} P_{i j} d V
$$

where $P_{i j}$ is the first Piola-Kirchhoff stress, instead of the Cauchy stress in Eq. 2.3. Fixing the first Piola-Kirchhoff stress imposes a constant dead load on the simulation cell.

Note that both deformation and stress constraints can be applied simultaneously provided they are imposed in different directions (i.e. the indices $i j$ are different). In addition, the cell rigid body translation and rotations must be removed, as described in further detail below.

\subsection{Enforcing cell average and Hill-Mandel conditions}

\subsubsection{Previous work}

A previous version of the ANL model, embedded in a different solver (WARP3D, http:// www. warp3d.net/) implemented the cell average and Hill-Mandel constraints with a unified approach, described in [9]. The idea is to enforce face-face constraints of the type:

$$
\mathbf{u}_{+}-\mathbf{u}_{-}=(\hat{\mathbf{F}}-\mathbf{I}) \cdot\left(\mathbf{X}_{+}-\mathbf{X}_{-}\right)
$$

where $\mathbf{X}_{+}$and $\mathbf{X}_{-}$are the coordinates of the periodic faces in the undeformed configuration. The trick in this approach is to let the deformation conditions be extra, dummy degrees of freedom, often implemented as dummy nodes in the finite element mesh:

$$
u_{i j}^{\star}=F_{i j}-\delta_{i j} .
$$

With this formulation, applying Dirichlet boundary conditions to the dummy degrees of freedom imposes cell-average deformation conditions, as Eq. 2.6 directly suggests, while 
imposing Neumann boundary conditions applies components of the volume-integrated first Piola-Kirchhoff stress, i.e. $\int_{V} P_{i j} d V$.

The WARP3D implementation imposed Eq. 2.6 using multipoint constraints. Note the conditions are true multipoint constraints as they will generally involve displacement components from the two faces and the dummy degrees of freedom. The current MOOSE constraint system is unsuited for implementing these types of conditions in 3D and so we examined other approaches. These conditions can be rephrased as Lagrange multiplier constraints imposed on nonlinear equations describing the unconstrained cell. In theory, there is no reason why this implementation should not work in MOOSE, using scalar kernels to impose the Lagrange multiplier equations. However, a trial ANL implementation was not numerically stable, and so we considered a third option.

\subsubsection{New formulation}

A superposition of conditions which individually meet the Hill-Mandel constraint also meets the constraint. One way to enforce a combination of cell-average conditions and the HillMandel condition is to divide the displacement field into two components:

$$
u_{i}=u_{i}^{\mu}+u_{i}^{M}
$$

where the microdisplacement field $\left(u_{i}^{\mu}\right)$ obeys periodic boundary conditions and the macrodisplacement field $\left(u_{i}^{M}\right)$ is affine with some imposed deformation, i.e.

$$
u_{i}^{M}=G_{i J} X_{J} .
$$

The constant deformation $G_{i J}$ represents 6 (3D, small deformation theory) or 9 (3D, large deformation theory) extra degrees of freedom. These extra degrees of freedom correspond to the extra constraint equations required to set the cell average stress or deformation conditions.

MOOSE already implements a version of this scheme to impose homogenization conditions on cell simulations for small deformation kinematics. Unfortunately, extending this implementation to large deformation kinematics is not trivial.

In theory, the additions to the MOOSE material and tensor mechanics kernel system are straightforward. Whenever the gradient of the displacements

$$
u_{i, J}
$$

appears replace it with the expression

$$
u_{i, J}=u_{i, J}^{\mu}+G_{i J}^{m}
$$

where $G_{i J}^{m}$ is a constant tensor field, represented with an appropriate number of ScalarVariables in MOOSE. Note the deformation gradient simply becomes

$$
F_{i J}=\delta_{i J}+u_{i, J}=\delta_{i J}+u_{i, J}^{\mu}+G_{i J}^{m} .
$$

The implementation supplements this addition to the model kinematics with the appropriate number of cell-average constraints. Deformation and stress constraints can be mixed and 


\begin{tabular}{l|ccc}
\hline \multirow{2}{*}{ Kinematics } & \multicolumn{3}{|c}{ Dimension } \\
& 1 & 2 & 3 \\
\hline Small & 1 & 3 & 6 \\
Large & 1 & 4 & 9 \\
\hline
\end{tabular}

Table 2.1: Number of extra scalar degrees of freedom and corresponding cell average constraints required for different cell formulations.

matched, provided they are imposed on different components of the cell-average quantities. These constraints are imposed using MOOSE ScalarKernels with the residuals:

$$
\begin{aligned}
& \hat{R}_{i j}=\frac{1}{V} \int_{V} F_{i J} d V-\hat{F}_{i J}=0 \\
& \hat{R}_{i j}=\frac{1}{V} \int_{V} P_{i J} d V-\hat{P}_{i J}=0
\end{aligned}
$$

where the first Piola-Kirchhoff stress can be calculated from the deformation gradient and the Cauchy stress

$$
P_{i J}=J \sigma_{i k} F_{J k}^{-1} \text {. }
$$

These constraints, in addition to periodic and rigid body constraints on the microdisplacement field $\left(u_{i}^{\mu}\right)$, are sufficient to fully-constrain the cell.

This framework works for 1D, 2D, and 3D models and for either small deformation or large deformation kinematics. The only changes are the dimension of the extra gradient tensor, $G_{i J}^{m}$, and the difference between a symmetric gradient (small deformations) or an unsymmetric gradient (large deformations). Table 2.1 outlines the required number of extra degrees of freedom, corresponding to the Cartesian entries in the appropriate type of $G_{i J}^{m}$ tensor, which equals the number of required cell-average constraints.

In MOOSE, we implemented the additional gradient term in the strain calculator, updated the Jacobian terms, added the extra degrees of freedom for the extra gradient as ScalarVariables, and imposed the cell average constraints as ScalarKernels (with the appropriate on- and off-diagonal Jacobian terms). In practice, the constraints are easier to implement as simple volume integrals, i.e. constraints of the form

$$
R=\frac{1}{V} \int_{V} C d V-\hat{C}=0
$$

can be rewritten as

$$
R=\int_{V}(C-\hat{C}) d V=0 .
$$

This changes the interpretation of the constraint residual, but not the final constraint.

\subsubsection{Problems with the basic formulation}

Thus far, the formulation described here could be implemented with minimal changes to the existing MOOSE small deformation system for imposing homogenization constraints. However, there are several implementation challenges. 


\subsubsection{Interface formulation}

In addition to the standard large deformation solid mechanics, the creep simulations include an interface model implemented in the MOOSE Discontinuous Galerkin system. This interface kernel takes as input the displacement jump across some internal interface:

$$
\llbracket u_{i} \rrbracket=u_{i}^{l e f t}-u_{i}^{r i g h t}
$$

and reports the corresponding interface traction. However, recall that we impose the cell average constraints using an extra gradient term and not the corresponding affine displacement field. How does this affect the interface formulation, which works with displacements and not gradients?

In the end, the gradient-based implementation does not affect interface formulations of this type. By definition the macro displacement field is affine, which means

$$
\llbracket u_{i}^{m} \rrbracket=0
$$

for any interface in the simulation. This implies that any interface displacement jumps are part of the microdisplacement field. These displacements feed the interface formulation and so the interface model remains consistent with the Hill-Mandel conditions and the macroscale cell average constraints.

\subsubsection{Rigid body modes}

Rigid body modes can exist in both the macro- and microdisplacement fields. The user must remove the rigid translation and rotation modes for the microdisplacement field manually, for example by constraining a sufficient number of nodal displacements to eliminate rigid translation and rotation.

The macrodisplacement field does not admit rigid translations, as it arises from the extra gradient term. However, for large deformation kinematics it may contain rigid rotations in $2 \mathrm{D}$ or $3 \mathrm{D}$. These can be removed by constraining the deformation represented by one of the single (2D) or three (3D) off diagonal pairs

\subsubsection{Mechanics kernel}

The MOOSE mechanics system uses an updated Lagrangian formulation. This approach formulates the equilibrium conditions in the current configuration and relies on calculating gradients with respect to the current coordinates by using the updated coordinates

$$
\mathbf{x}=\mathbf{X}+\mathbf{u}
$$

This type of formulation uses the MOOSE use_displaced_mesh option to maintain the appropriate updated geometry. However, when applying the homogenization constraints using the extra gradient term the affine displacements are not calculated or included in the displacement field MOOSE uses to update the current coordinates. This means the kernel will not include the effect of the constant gradient field in calculating the equilibrium conditions in the current frame, nor will displacements with respect to the current coordinates include the effect of the extra gradient term. These issues must be corrected to have a fullyconsistent, large-displacement homogenization system. 


\subsection{Total Lagrangian kernel}

We elected to rewrite the MOOSE large displacement solid mechanics kernel system to refer only to the reference configuration (i.e. a total Lagrangian formulation). This eliminates the need for the use_displaced_mesh flag and means that the effects of the extra homogenization gradient term are included in the kinematic formulation.

\subsubsection{Residual equation}

The residual equation for the total Lagrangian formulation is

$$
R^{\alpha}=\int_{V} J \sigma_{i j} \phi_{i, K}^{\alpha} F_{K j}^{-1} d V
$$

where $J=\operatorname{det} \mathbf{F}$ and $\phi$ are the test functions. This formulates the equilibrium conditions in the undeformed configuration and only requires gradients with respect to the initial coordinates. Therefore, the total Lagrangian kernel will integrate correctly with the homogenization described above.

It is more common when working with a total Lagrangian formulation to note that

$$
J \sigma_{i j} F_{K j}^{-1}=P_{i K}
$$

and so

$$
R^{\alpha}=\int_{V} P_{i K} \phi_{i, K}^{\alpha} d V=0
$$

and then make the constitutive model system responsible for returning the first PiolaKirchhoff stress instead of the Cauchy stress. However, both the NEML and MOOSE constitutive model systems return the Cauchy stress and so our implementation keeps the longer, Cauchy stress form of Eq. 2.21. This means that the material systems for the original, updated formulation and the new, total formulation are the same. Two otherwise-identical problems using the two different kernel approaches should produce exactly the same results.

\subsubsection{Jacobian terms}

In the following $\Upsilon^{\beta}$ are the discrete nodal displacements and $\psi$ are the trial functions.

The Jacobian has three terms:

$$
\frac{\partial R^{\alpha}}{\partial \Upsilon^{\beta}}=\int_{V}\left(\frac{\partial J}{\partial \Upsilon^{\beta}} \sigma_{i j} \phi_{i, K}^{\alpha} F_{K j}^{-1}+J \frac{\partial \sigma_{i j}}{\partial \Upsilon^{\beta}} \phi_{i, K}^{\alpha} F_{K j}^{-1}+J \sigma_{i j} \phi_{i, K}^{\alpha} \frac{\partial F_{K j}^{-1}}{\partial \Upsilon^{\beta}}\right) d V
$$

which we label for convenience:

$$
J^{\alpha \beta}=\int_{V} A^{\alpha \beta}+B^{\alpha \beta}+C^{\alpha \beta} d V
$$

Working term-by-term:

$$
A^{\alpha \beta}=J F_{L k}^{-1} \psi_{k, L}^{\beta} \sigma_{i j} \phi_{i, K}^{\alpha} F_{K j}^{-1}
$$




$$
B^{\alpha \beta}=J C_{i j m n} F_{m A}^{(n)} F_{A s}^{-1} F_{T n}^{-1} \psi_{s, T}^{\alpha} \phi_{i, K}^{\alpha} F_{K j}^{-1}
$$

which we could write

$$
B^{\alpha \beta}=J C_{i j m n} f_{m s}^{-1} F_{T n}^{-1} \psi_{s, T}^{\alpha} \phi_{i, K}^{\alpha} F_{K j}^{-1}
$$

and finally

$$
\begin{gathered}
C^{\alpha \beta}=J \sigma_{i j} \phi_{i, K}^{\alpha} \frac{\partial F_{K j}^{-1}}{\partial F_{m N}} \frac{\partial F_{m N}}{\partial \Upsilon^{\beta}}=J \sigma_{i j} \phi_{i, K}^{\alpha} \frac{\partial F_{K j}^{-1}}{\partial F_{m N}} \psi_{m, N}^{\beta} \\
C^{\alpha \beta}=-J \sigma_{i j} \phi_{i, K}^{\alpha} F_{K m}^{-1} F_{N j}^{-1} \psi_{m, N}^{\beta}
\end{gathered}
$$

We can combine the first and last terms and make simplifications:

$$
\begin{gathered}
A^{\alpha \beta}+C^{\alpha \beta}=J F_{L k}^{-1} \psi_{k, L}^{\beta} \sigma_{i j} \phi_{i, K}^{\alpha} F_{K j}^{-1}-J \sigma_{i j} \phi_{i, K}^{\alpha} F_{K m}^{-1} F_{N j}^{-1} \psi_{m, N}^{\beta} \\
A^{\alpha \beta}+C^{\alpha \beta}=J \phi_{i, K}^{\alpha} F_{K j}^{-1} \sigma_{i j} \psi_{k, N}^{\beta} F_{N k}^{-1}-J \phi_{i, K}^{\alpha} F_{K m}^{-1} \sigma_{i j} \psi_{m, N}^{\beta} F_{N j}^{-1} \\
A^{\alpha \beta}+C^{\alpha \beta}=J \sigma_{i j}\left(\psi_{i, M}^{\beta} F_{M j}^{-1} \phi_{k, N}^{\alpha} F_{N k}^{-1}-\psi_{i, M}^{\beta} F_{M k}^{-1} \phi_{k, N}^{\alpha} F_{N j}^{-1}\right) \\
A^{\alpha \beta}+C^{\alpha \beta}=J \psi_{i, M}^{\beta} \sigma_{i j} \phi_{k, N}^{\alpha}\left(F_{M j}^{-1} F_{N k}^{-1}-F_{M k}^{-1} F_{N j}^{-1}\right)
\end{gathered}
$$

If we define:

$$
\begin{aligned}
& \Phi_{i, j}^{\alpha}=\phi_{i, k}^{\alpha} F_{k, j}^{-1} \\
& \Psi_{i, j}^{\beta}=\psi_{i, k}^{\beta} F_{k, j}^{-1}
\end{aligned}
$$

then we have:

$$
\begin{aligned}
B^{\alpha \beta} & =J C_{i j m n} f_{m k}^{-1} \Psi_{k, n}^{\beta} \Phi_{i, j}^{\alpha} \\
A^{\alpha \beta}+C^{\alpha \beta} & =J \sigma_{i j}\left(\Psi_{i, j}^{\beta} \Phi_{k, k}^{\alpha}-\Psi_{i, k}^{\beta} \phi_{k, j}^{\alpha}\right)
\end{aligned}
$$

\subsubsection{Verification}

As noted above, the updated and total Lagrangian kernels should produce identical results for identical problems. Defining a verification case is straightforward as the two models can use the same materials, boundary conditions, etc.

Figure 2.2 presents a simple verification case. The simulation deforms a 3D block of material into a $\pi / 4$ angle bend. The material model is a simple rate-independent elasticplastic model with Voce isotropic hardening. Subfigures (a) and (b) show the deformed geometry at the same time for the current updated Lagrangian and new total Lagrangian formulations. Subfigure (c) plots the total dissipated work as a function of time, again for the updated and total Lagrangian formulations. Subfigures (a) and (b) are identical and the two lines in (c) exactly overlap, verifying the total Lagrangian kernel. 


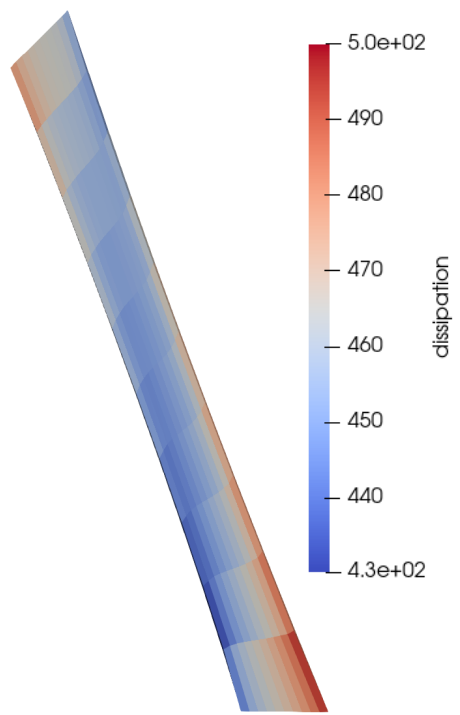

(a)

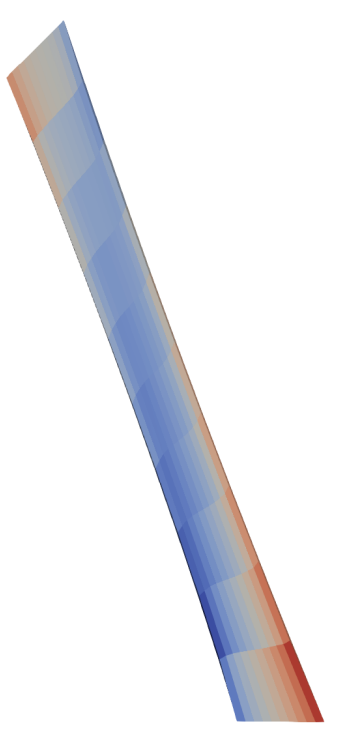

(b)

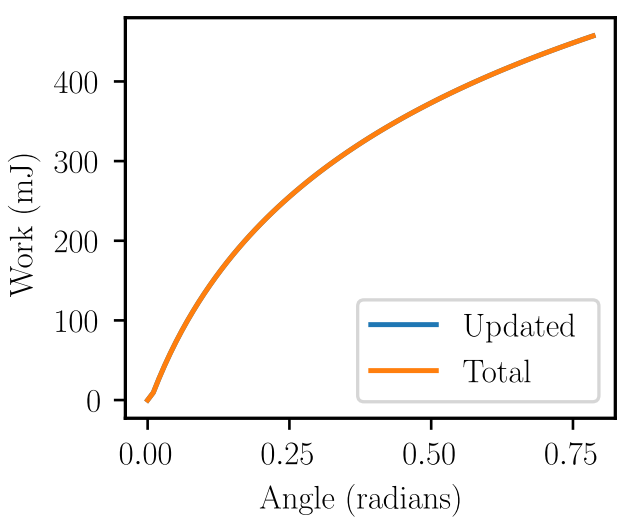

(c)

Figure 2.2: Comparison between the updated and total Lagrangian formulations for a simple forming problem. (a) Deformed shape for the updated Lagrangian formulation. (b) Deformed shape for the total Lagrangian formulation. (c) Total dissipated energy (work) plotted as a function of bend angle for the two formulations. The two lines exactly overlap.

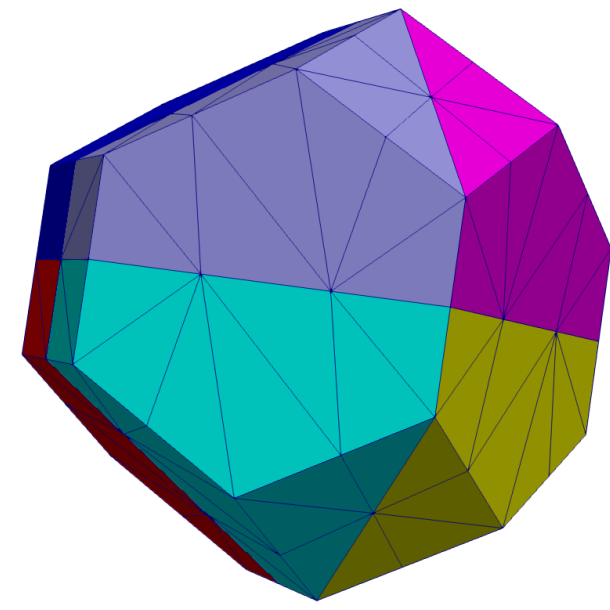

(a)

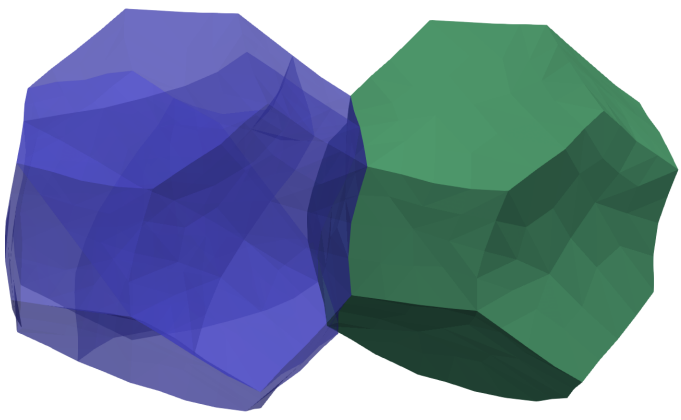

(b)

Figure 2.3: (a) Simple truncated octahedron cell for the verification calculation. (b) Demonstration that the cell remains periodic after significant deformation. 


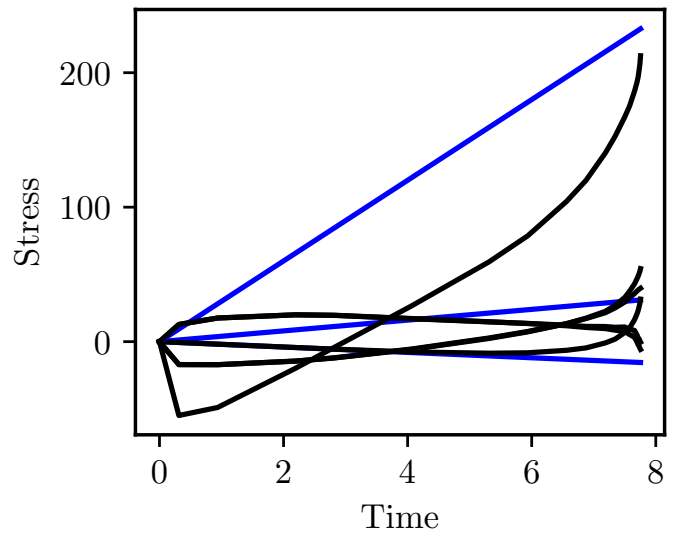

(a) First Piola-Kirchhoff stress.

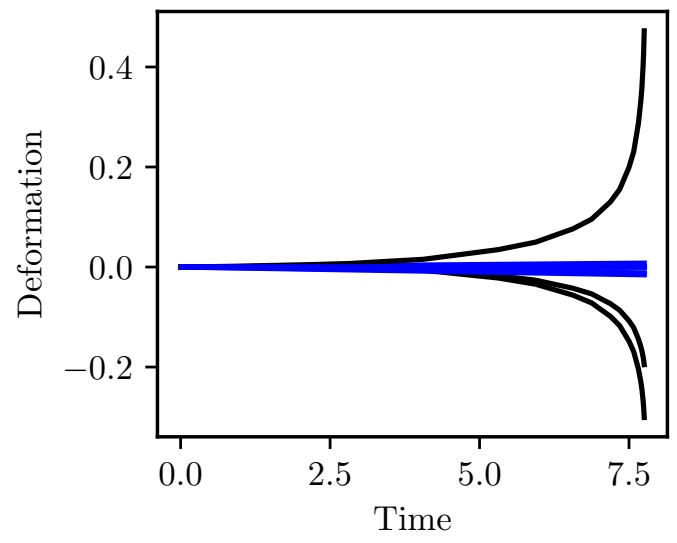

(b) Deformation

Figure 2.4: Plots of the cell-average stress and deformation, demonstrating that the model obeys the imposed constraints.

\subsection{Verification}

Figure 2.3(a) shows a simple periodic cell consisting of 8 randomly oriented grains. The cell geometry is simple, but deliberately setup to not have simple Cartesian periodicity. The 3D model applies the homogenization system for large deformations with mixed constraints: three zero deformation constraints to remove rigid rotations, three average stress constraints, and three average deformation constraints. Figure 2.3(b) shows the deformed unit cell and demonstrates that after deformation the cell remains periodic. Note that only the microdisplacements appear in the deformed volume rendering, as the macrodisplacements are never explicitly calculated. However, the macrodisplacement field is clearly periodic as it is affine with the imposed gradient.

Finally, Figures 2.4(a) and (b) demonstrate that the average stress and deformation conditions match the applied values. Subfigure (a) plots the cell-average 1st Piola-Kirchhoff stress; subfigure (b) plots the average deformation $(\mathbf{F}-\mathbf{I})$. The constrained values appear as black lines in the diagrams. These were ramped linearly. The figures plot the uncon-

strained values in blue. The cell averages match the constrained values, thus verifying the homogenization system. 


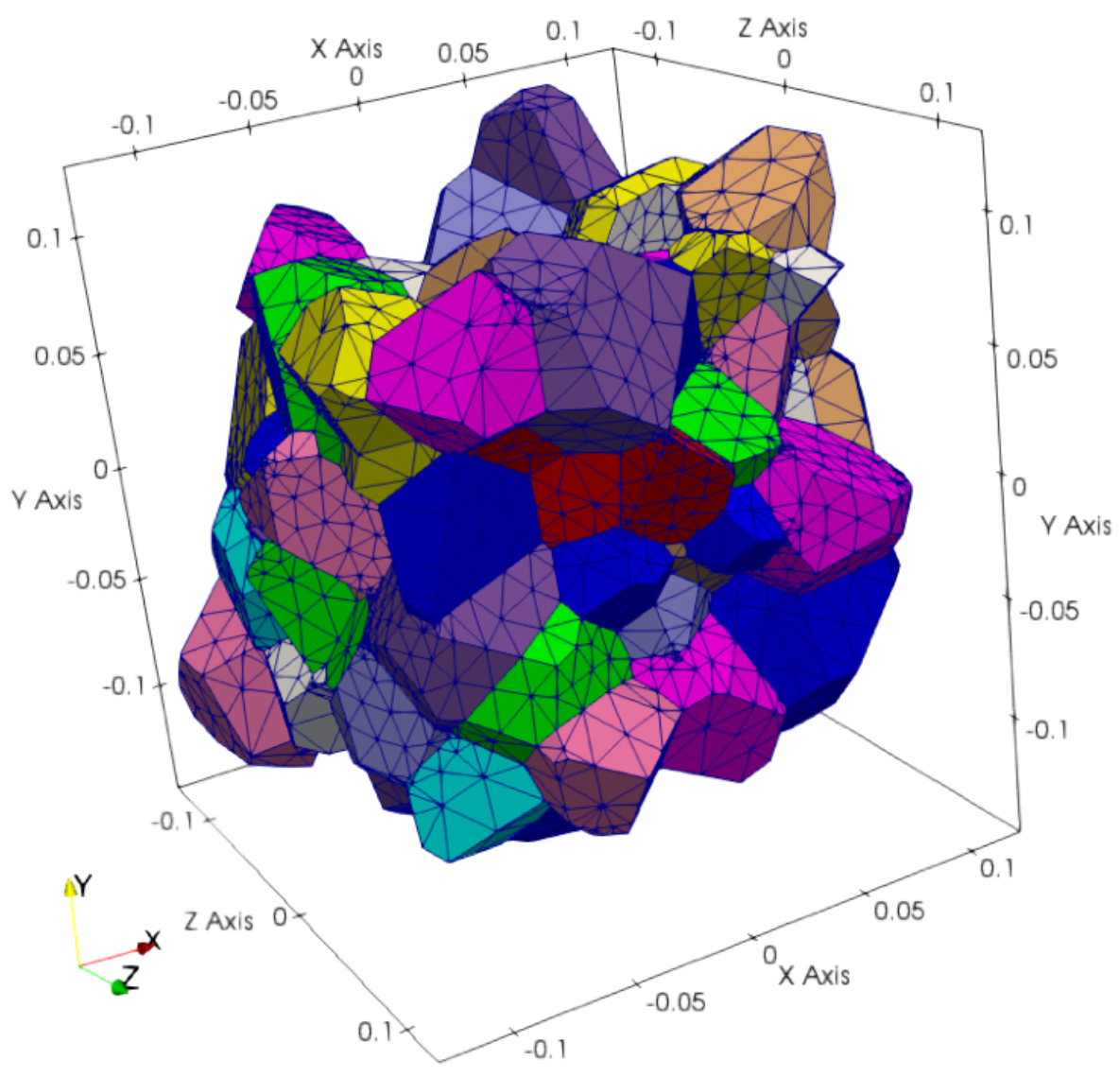

Figure 2.5: Render of a synthetic periodic microstructures with 100 grains, generated with Neper, colored by grain number.

\subsection{Identification of periodic boundaries}

MOOSE enforces periodic boundary conditions by imposing periodicity of a variable value on periodic domain boundaries. For a rectangular cuboid cell MOOSE can identify the six periodic cell boundaries, and automatically choose the correct periodic pairs. However, for more complex periodic cell types the automatic periodic boundary detection feature can not be used. Neper [6] generates complex periodic cell shapes like the one depicted in Figure 2.5 .

Furthermore, the periodic unit cells generated by Neper possess multiple periodic, nonflat, surfaces which can be characterized by the following families of translation directions:

- $\langle 001\rangle$,

- $\langle 011\rangle$,

- $\langle 111\rangle$.

The set of of all translation vector is obtained by applying the 24 cubic symmetries to all the translation vector families and retaining the linearly independent set. This operation 
generates a set of 13 independent translation vector. Each translation vector is associated to a pair of periodic, non-flat surfaces. In finite elements, a surface is represented by a collection of element sides. Each element side is characterized by a set of nodes. Therefore, given a specific translation direction, $d$ two sides, $s_{1}$ and $s_{2}$, are periodic if the nodes describing them are translated by a distance $d$.

Given the above, a pseudo algorithm to identify periodic boundaries is the following:

1. find all the nodes on the surface of the periodic cell

2. for each node $i$ loop over all the nodes, $j$, and check if they are periodic for any of the thirteen translation vector $d_{k}$. If the node pair is periodic for direction $d_{k}$, add node $i$ to the list of nodes associated to the primary side of $d_{k}$, and add node $j$ to the list of nodes associated the secondary side of $d_{k}$. This process will generate a set of twenty six node sets.

3. for each node set, identify all the element-sides belonging to it and add them the associated side-set.

Neper can generate periodic meshes that can be directly used as an input for MOOSE. Therefore to automate the process of creating periodic boundaries we develop a mesh generator in MOOSE that identifies periodic boundaries, and automatically set all the required periodic boundary conditions. Figure 2.6 is an example showing the identified periodic, non-flat boundaries.

We plan to merge this tool into MOOSE proper. 


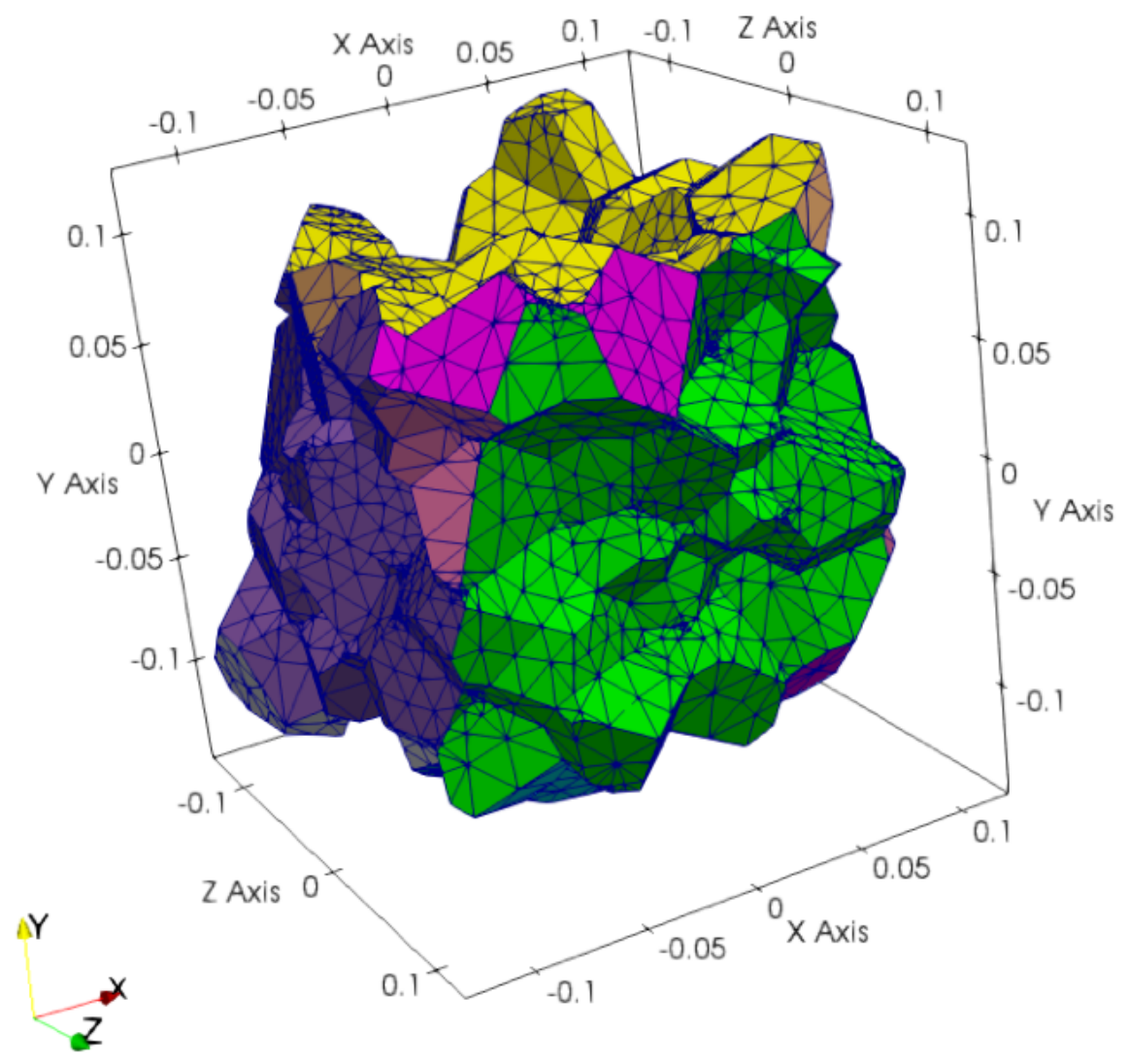

Figure 2.6: Render of a synthetic periodic microstructures highlighting the identified periodic surfaces. Different colors are associated to different periodic surfaces. 



\section{Large deformation cohesive zone model in MOOSE}

Libmesh [10], which is the finite element library on which MOOSE is built on [11], does not support conventional interface elements. Therefore Messner et al. [12] utilized an elementless discontinuous Galerkin approach for cohesive zone modeling in MOOSE. Rovinelli et al. [13] successfully used this method to perform physics-based crystal plasticity simulation, including grain boundary cavitation, to investigate the ability of different effective stress measures to predict the creep life of Grade 91 steel. However, the cohesive zone model currently available in MOOSE does not account for large interface area changes and rotations. Creep rupture in ductile materials, such as Grade 91, occurs with area reduction factors grater than $80 \%$ [14], thus justifying the need for large deformation cohesive zone model in MOOSE.

To implement any large deformation constitutive model the deformation gradient $F$ must be known. In general $F$ can be computed at any integration point by using nodal displacements and shape functions. Standard cohesive zone modeling is not an exception as it relies on the knowing $F$ on the cohesive interface. The cohesive interface is generally modeled with cohesive elements [15]. Cohesive elements are a special type of zero-thickness elements providing integration points on their mid-plane. Most large deformation implementations use the mid-plane to define the cohesive separation law, which carries over to discontinuous Galerkin approaches [16]. These implementations also enforce traction equilibrium on the midplane.

However, the MOOSE DG formulation integrates over the element sides, which is a challenge for large deformations. The lack of integration points on the mid-plane forced us to use an assumption to recover the deformation gradient on the interface mid-plane. As a first order approximation we assume the deformation gradient on the mid-plane, $F^{*}$, to be the average of the deformation gradient on the + and - surfaces:

$$
F^{*}=\frac{F^{+}+F^{-}}{2} .
$$

Figure 3.1 is a schematic representing the cohesive interface, *, and the surfaces of the two elements generating the cohesive interface together with the available quadrature points.

For large deformation problems, MOOSE allows equilibrium to be imposed on both the deformed or the undeformed mesh. When solving a solid mechanics problem one has to satisfy both the linear and angular momentum equilibrium. For equilibrium applied on the undeformed configuration angular momentum is automatically satisfied because of the zero thickness interface assumption. If the formulation imposes equilibrium in the deformed configuration angular momentum conservation must be enforced with constraints on the cohesive constitutive model. Note the analogy here with conventional solid mechanics: total Lagrangian formulations impose equilibrium in the undeformed configuration working with the 1st Piola-Kirchhoff stress, which is a general rank 2 tensor. Updated Lagrangian formulations enforce equilibrium in the deformed configuration but the constitutive model must maintain a symmetric Cauchy stress to conserve angular momentum. We elected to use a total Lagrangian formulation. The total Lagrangian formulation requires a map between the first Piola-Kirchoff traction, $T$, the Cauchy's traction $t$ and the infinitesimal force $d f$. The following relationship defines the equivalence between, $d f, T$ and $t$ :

$$
d f=T d S=t d s
$$




\section{undeformed deformed}

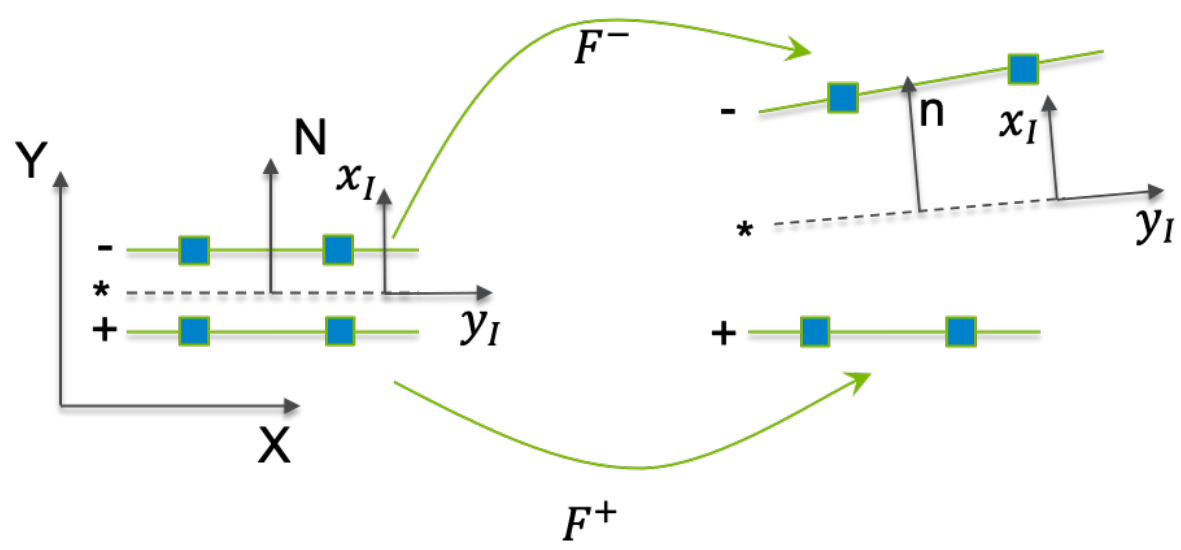

Figure 3.1: Two dimensional schematic depicting the three surface, denoted by,+- and * symbols, used in the large deformation cohesive zone model along with the locations of the available integration points, e.g. blue squares. The + and - surfaces are the surfaces of two adjacent elements defining the cohesive interface. The $*$ surface represents the cohesive interface mid-plane. Notice that, the,+- and $*$ surfaces are coincident in the undeformed configuration but they are drawn as separated for illustration purposes.

where $d S$ is the infinitesimal area in the undeformed configuration, and $d s$ is the infinitesimal area in the deformed configuration. Both $d S$ and $d s$ are scalars in Equation 3.2.

The relationship between the first Piola-Kirchoff traction and the Cauchy's traction can be rewritten as

$$
T=t \frac{d s}{d S}
$$

The objective traction rate is obtained by taking the time derivative of Equation 3.3:

$$
\dot{T}=\frac{1}{d S}(d \dot{s} t+d s \dot{t})
$$

Constitutive cohesive material models are defined as the normal and shear traction rate as function of the normal and shear displacement jump rates, $t^{I}\left(\llbracket \dot{u} \rrbracket^{I}\right)$. However, equilibrium is imposed using a traction defined in the global reference system. Therefore a transformation between the interface and the global coordinate system must be specified. In the undeformed configuration, the interface coordinate system can be defined as:

$$
Q_{0}=\left[N, S_{1}, S_{2}\right]
$$

where $N$ is the interface normal in the undeformed configuration, and $S_{1}$ and $S_{2}$ are two arbitrary orthogonal directions in the interface plane.

To account for large rotations, the rotation of the interface mid-plane must be considered. By realizing that the interface coordinate system rigidly rotates following the interface midplane, the total rotation matrix required to transform a vector from the interface to the global coordinate system is:

$$
Q=Q_{0} R^{T}
$$


where $R$ is the rotation matrix accounting for the interface mid-plane rotation. The rotation matrix $Q$ can be used to rotate the displacement jump and the traction from the deformed interface coordinate system to the global coordinate system as:

$$
\begin{aligned}
\llbracket u \rrbracket & =Q^{T} \llbracket u \rrbracket^{I} . \\
t & =Q^{T} t^{I} .
\end{aligned}
$$

By differentiating Eq. 3.7 and 3.8 with respect to time one obtains:

$$
\begin{aligned}
\llbracket \dot{u} \rrbracket & =\dot{Q}^{T} \llbracket u \rrbracket^{I}+Q^{T} \llbracket \dot{u} \rrbracket^{I} . \\
\dot{t} & =\dot{Q}^{T} t^{I}+Q^{T} \dot{t}^{I} .
\end{aligned}
$$

Finally, substituting 3.8 and 3.10 in Eq. 3.4 one obtains:

$$
\dot{T}=\frac{d s}{d S}\left(\left(\frac{d \dot{s}}{d s} Q^{T}+\dot{Q}^{T}\right) t^{I}+Q^{T} \dot{t}^{I}\right) .
$$

Notice that Eq. 3.11 reduces to $\dot{T}=Q^{T} \dot{t}^{I}=\dot{t}$ when large rotation and area changes are neglected (e.g., when $d \dot{s}=\dot{Q}^{T}=0$ and $\frac{d s}{d S}=1$ ), which is a consistency check. Equation 3.11 embeds all the kinematics, thus decoupling the kinematics from the traction-separation constitutive equation. The traction separation constitutive equation is assumed to be defined in the interface mid-plane in the deformed configuration.

Decoupling the kinematics and constitutive equations greatly eases the implementation of custom traction separation laws because the only terms that need to be defined are:

- the material constitutive rate equations in the interface coordinate system and $\dot{t}^{I}\left(\llbracket \dot{u} \rrbracket^{I}\right)$,

- the total derivatives of interface traction rates with respect to the interface displacement jump, $\frac{d \dot{t}_{i}^{I}}{d \llbracket \dot{u} \rrbracket_{j}^{I}}$.

Equation 3.11 was implemented in the MOOSE cohesive zone model material system using the following incremental formulation:

$$
\Delta T=\frac{d s}{d S}\left(\left(\frac{\Delta d s}{d s} Q^{T}+\Delta Q^{T}\right) t^{I}+Q^{T} \Delta t^{I}\right) .
$$

\subsection{Implementation in MOOSE}

\subsubsection{Cohesive model residual}

The residual equation for the total Lagrangian cohesive zone formulation for the displacement component $i$ can be written as:

$$
\begin{aligned}
& R_{i}^{\alpha,+}=-\int_{S} T_{i}(\llbracket u \rrbracket)_{i} \phi_{i}^{\alpha,+} d S \\
& R_{i}^{\alpha,-}=\int_{S} T_{i}(\llbracket u \rrbracket)_{i} \phi_{i}^{\alpha,-} d S
\end{aligned}
$$


where $\alpha$ is the test function index and $\phi^{+}$and $\phi^{-}$are the test function associated to the + and - surfaces, respectively. Notice that in the above equation $i$ is not a summation index, but represents the coordinate $i$.

\subsubsection{Cohesive model Jacobian}

The Jacobian is the derivative of the residual with respect to the discrete displacements $\Upsilon^{\beta}$. The traction on each side of the interface is a function of the displacement jump, $u \rrbracket$, therefore the residual on both element faces is function of the displacement on both sides of interface. This then requires the derivatives

$$
\begin{aligned}
& \frac{d R_{i}^{\alpha,+}}{d \Upsilon_{j}^{\beta,+}}=-\int_{S} \frac{d T_{i}(\llbracket u \rrbracket) \phi_{i}^{\alpha,+}}{d \Upsilon_{j}^{\beta,+}} d S=-\int_{S} \frac{d \Delta T_{i}(\llbracket u \rrbracket)}{d \Upsilon_{j}^{\beta,+}} \phi_{i}^{\alpha,+} d S \\
& \frac{d R_{i}^{\alpha,+}}{d \Upsilon_{j}^{\beta,-}}=-\int_{S} \frac{d T_{i}(\llbracket u \rrbracket) \phi_{i}^{\alpha,+}}{d \Upsilon_{j}^{\beta,-}} d S=-\int_{S} \frac{d \Delta T_{i}(\llbracket u \rrbracket)}{d \Upsilon_{j}^{\beta,-}} \phi_{i}^{\alpha,+} d S \\
& \frac{d R_{i}^{\alpha,-}}{d \Upsilon_{j}^{\beta,+}}=\int_{S} \frac{d T_{i}(\llbracket u \rrbracket) \phi_{i}^{\alpha,-}}{d \Upsilon_{j}^{\beta,+}} d S=\int_{S} \frac{d \Delta T_{i}(\llbracket u \rrbracket)}{d \Upsilon_{j}^{\beta,+}} \phi_{i}^{\alpha,+} d S \\
& \frac{d R_{i}^{\alpha,-}}{d \Upsilon_{j}^{\beta,-}}=\int_{S} \frac{d T_{i}(\llbracket u \rrbracket) \phi_{i}^{\alpha,-}}{d \Upsilon_{j}^{\beta,-}} d S=\int_{S} \frac{d \Delta T_{i}(\llbracket u \rrbracket)}{d \Upsilon_{j}^{\beta,-}} \phi_{i}^{\alpha,+} d S
\end{aligned}
$$

In writing Eqs. 3.16 - 3.19 we have implicitly noticed that: i) $d S$ is constant, ii) the test functions $\phi^{\alpha}$ are independent from the discrete displacements, iii) $T_{i}=T_{\text {old }, i}+\Delta T_{i}$, and iv) $T_{\text {old }, i}$ is independent from the discrete displacements.

We start by recalling the finite element discretization for a variable:

$$
u \approx u_{h}=\sum_{\beta} \Upsilon^{\beta} \psi^{\beta}
$$

where $\psi$ indicates the trial function. By utilizing the displacement jump definition and the finite element discretization, the displacement jump for the coordinate $i$ can be expressed as:

$$
\llbracket u \rrbracket_{i}=u_{i}^{-}-u_{i}^{+} \approx \sum_{\beta,-} \Upsilon_{i}^{\beta,-} \psi_{i}^{\beta,-}-\sum_{\beta,+} \Upsilon_{i}^{\beta,+} \psi_{i}^{\beta,+}
$$

The derivative of the displacement jump with respect to the discrete displacements can be approximated as:

$$
\begin{aligned}
& \frac{d \llbracket u \rrbracket_{i}}{d \Upsilon_{j}^{\beta,-}} \approx \psi_{i}^{\beta,-} \quad \text { if } i=j, \text { else } 0 \\
& \frac{d \llbracket u \rrbracket_{i}}{d \Upsilon_{j}^{\beta,+}} \approx-\psi_{i}^{\beta,+} \quad \text { if } i=j, \text { else } 0
\end{aligned}
$$


where $\delta_{i j}$ is the Kronecker delta. By noticing that the deformation gradients can be approximated as:

$$
\begin{aligned}
& F_{i j}^{+} \approx \delta_{i j}+\sum_{\beta} \Upsilon_{i}^{\beta,+} \nabla \psi_{i j}^{\beta,+} \\
& F_{i j}^{-} \approx \delta_{i j}+\sum_{\beta} \Upsilon_{i}^{\beta,-} \nabla \psi_{i j}^{\beta,-}
\end{aligned}
$$

where $\delta_{i j}$ is the Kronecker delta. By using equation 3.1 the derivatives of $F^{*}$ with respect to the discrete displacements becomes:

$$
\begin{aligned}
\frac{d F_{i j}^{*}}{d \Upsilon_{k}^{\beta,-}} & \approx \frac{1}{2} \nabla \psi_{i j}^{\beta,-} \quad \text { if } k=i, \text { else } 0 \\
\frac{d F_{i j}^{*}}{d \Upsilon_{k}^{\beta,+}} & \approx \frac{1}{2} \nabla \psi_{i j}^{\beta,+} \quad \text { if } k=i, \text { else } 0
\end{aligned}
$$

To simplify the Jacobian development, we define the following quantities:

$$
\begin{aligned}
A & =\frac{d s}{d S} \\
B & =\left(\frac{\Delta d s}{d s} Q^{T}+\Delta Q^{T}\right) \\
C & =Q^{T}
\end{aligned}
$$

and use them to rewrite Eq. 3.12 as:

$$
\Delta T=A\left(B t^{I}+C \Delta t^{I}\right)
$$

From Eqs. 3.16 - 3.19 is apparent that computing the Jacobian implies computing the following derivatives:

$$
\begin{aligned}
& \frac{d \Delta T_{i}(\llbracket u \rrbracket)}{d \Upsilon_{j}^{\beta,+}}=\frac{d}{d \Upsilon_{j}^{\beta,+}}\left(A\left(B_{i k} t_{k}^{I}+C_{i k} \Delta t_{k}^{I}\right)\right) \\
& \frac{d \Delta T_{i}(\llbracket u \rrbracket)}{d \Upsilon_{j}^{\beta,-}}=\frac{d}{d \Upsilon_{j}^{\beta,-}}\left(A\left(B_{i k} t_{k}^{I}+C_{i k} \Delta t_{k}^{I}\right)\right)
\end{aligned}
$$

By using the chain rule and doing some manipulation we obtain the following equations:

$$
\begin{gathered}
\frac{d \Delta T_{i}(\llbracket u \rrbracket)}{\partial \Upsilon_{j}^{\beta,+}}=\frac{\partial A}{d \Upsilon_{j}^{\beta,+}}\left(B_{i k} t_{k}^{I}+C_{i k} \Delta t_{k}^{I}\right)+A\left(\frac{\partial B_{i k}}{\partial \Upsilon_{j}^{\beta,+}} t_{k}^{I}+\frac{\partial C_{i k}}{\partial \Upsilon_{j}^{\beta,+}} \Delta t_{k}^{I}+\left(B_{i k}+C_{i k}\right) \frac{\partial \Delta t_{k}^{I}}{\partial \Upsilon_{j}^{\beta,+}}\right) \\
\frac{d \Delta T_{i}(\llbracket u \rrbracket)}{\partial \Upsilon_{j}^{\beta,-}}=\frac{\partial A}{d \Upsilon_{j}^{\beta,-}}\left(B_{i k} t_{k}^{I}+C_{i k} \Delta t_{k}^{I}\right)+A\left(\frac{\partial B_{i k}}{\partial \Upsilon_{j}^{\beta,-}} t_{k}^{I}+\frac{\partial C_{i k}}{\partial \Upsilon_{j}^{\beta,-}} \Delta t_{k}^{I}+\left(B_{i k}+C_{i k}\right) \frac{\partial \Delta t_{k}^{I}}{\partial \Upsilon_{j}^{\beta,-}}\right)
\end{gathered}
$$


The remaining partial derivatives of $A, B$, and $\Delta t^{I}$ can be computed by exploiting the chain rule again:

$$
\begin{aligned}
\frac{\partial A}{\partial \Upsilon_{j}^{\beta,+}} & =\frac{\partial A}{\partial F_{r s}^{*}} \frac{\partial F_{r s}^{*}}{\partial \Upsilon_{j}^{\beta,+}} & \frac{\partial A}{\partial \Upsilon_{j}^{\beta,-}} & =\frac{\partial A}{\partial F_{r s}^{*}} \frac{\partial F_{r s}^{*}}{\partial \Upsilon_{j}^{\beta,-}} \\
\frac{\partial B_{i k}}{\partial \Upsilon_{j}^{\beta,+}} & =\frac{\partial B_{i k}}{\partial F_{r s}^{*}} \frac{\partial F_{r s}^{*}}{\partial \Upsilon_{j}^{\beta,+}} & \frac{\partial B_{i k}}{\partial \Upsilon_{j}^{\beta,-}} & =\frac{\partial B_{i k}}{\partial F_{r s}^{*}} \frac{\partial F_{r s}^{*}}{\partial \Upsilon_{j}^{\beta,-}} \\
\frac{\partial C_{i k}}{\partial \Upsilon_{j}^{\beta,+}} & =\frac{\partial C_{i k}}{\partial F_{r s}^{*}} \frac{\partial F_{r s}^{*}}{\partial \Upsilon_{j}^{\beta,+}} & \frac{\partial C_{i k}}{\partial \Upsilon_{j}^{\beta,-}} & =\frac{\partial C_{i k}}{\partial F_{r s}^{*}} \frac{\partial F_{r s}^{*}}{\partial \Upsilon_{j}^{\beta,-}} \\
\frac{\partial \Delta t_{k}^{I}}{\partial \Upsilon_{j}^{\beta,+}} & =\frac{\partial \Delta t_{k}^{I}}{\partial \llbracket \Delta u \rrbracket_{r}^{I}} \frac{\partial \llbracket \Delta u \rrbracket_{r}^{I}}{\partial \llbracket u \rrbracket_{s}} \frac{\partial \llbracket u \rrbracket_{s}}{\partial \Upsilon_{j}^{\beta,+}} & \frac{\partial \Delta t_{k}^{I}}{\partial \Upsilon_{j}^{\beta,-}} & =\frac{\partial \Delta t_{k}^{I}}{\partial \llbracket \Delta u \rrbracket_{r}^{I}} \frac{\partial \llbracket \Delta \rrbracket_{r}^{I}}{\partial \llbracket u \rrbracket_{s}} \frac{\partial \llbracket u \rrbracket_{s}}{\partial \Upsilon_{j}^{\beta,-}}
\end{aligned}
$$

The partial derivatives of $F^{*}$ and $\llbracket u \rrbracket$ with respect to the discrete displacements have already been defined in Eqs. 3.22, 3.23, 3.27 and 3.28. The partial derivative of $t^{I}$ with respect to $\llbracket u \rrbracket^{I}$ is a material dependent derivative and will not be discussed here. By using Nanson's Formula and adopting other kinematics identities we can recast $A, B$ and $C$ as follows:

$$
\begin{aligned}
& A=\frac{d s}{d S}=\operatorname{det}(F)\left\|F^{-T} N\right\| \\
& B=\left(\frac{\Delta d s}{d s} Q^{T}+\Delta Q^{T}\right)=(\operatorname{trace}(L)-n \cdot(L n)) R Q_{0}^{T}+\Delta R Q_{0}^{T} \\
& C=Q^{T}=R Q_{0}^{T}
\end{aligned}
$$

where we dropped the superscript $*$ of $F$ to simplify the notation.

We start by computing $\frac{\partial C}{\partial F}$ as:

$$
\frac{\partial C_{i j}}{\partial F_{r s}}=\frac{\partial R_{i k}}{\partial F_{r s}} Q_{0, k j}^{T}
$$

where the partial derivative of the rotation matrix can be computed using the formula proposed by Chen and Wheeler [17]:

$$
\frac{\partial R_{k l}}{\partial F_{m n}}=\frac{R_{k p}}{\operatorname{det}(\bar{U})}\left(\bar{U}_{p q} R_{m q} \bar{U}_{n l}-\bar{U}_{p n} R_{m q} \bar{U}_{q l}\right)
$$

where $\bar{U}=\operatorname{trace}(U) I-U$. The incremental model also requires to compute $\frac{\partial \Delta R_{k l}}{\partial F_{m n}}$. In the implementation we used a linear rotation approximation therefore $\Delta R=R-R_{\text {old }}$. Noticing that $\frac{\partial R_{o l d, k l}}{\partial F_{m n}}=0$ we obtain

$$
\frac{\partial \Delta R_{i j}}{\partial F_{r s}}=\frac{\partial R_{i j}}{\partial F_{r s}}
$$

The next term we analyze is $\frac{\partial A}{\partial F}$ :

$$
\frac{\partial A}{\partial F_{p q}}=\frac{\partial \operatorname{det}(F)}{\partial F_{p q}}\left\|F^{-T} N\right\|+\operatorname{det}(F) \frac{\partial\left\|F^{-T} N\right\|}{F_{p q}}
$$


The derivative of the determinant is

$$
\frac{\partial \operatorname{det}(F)}{\partial F_{i j}}=\operatorname{det}(F) F_{i j}^{-T}
$$

The derivative of the norm can be computed by realizing that the derivative of the norm of a vector with respect to its component is:

$$
\frac{\partial\|V\|}{\partial V_{i}}=\frac{V_{i}}{\|V\|}
$$

and that the derivative of the inverse of a tensor with respect to its components is:

$$
\frac{\partial T_{i j}^{-}}{\partial T_{p q}}=T_{i p}^{-} T_{q j}^{-}
$$

By using Eqs. 3.49, 3.50 and 3.51 and substituting in Eq. 3.48 we obtain:

$$
\frac{\partial A}{\partial F_{p q}}=\operatorname{det}(F) F_{p q}^{-T}\left\|F^{-T} N\right\|+\operatorname{det}(F) \frac{F_{i k}^{-T} N_{k}}{\left\|F^{-T} N\right\|} F_{j p}^{-} F_{q i}^{-} N_{j}
$$

The other term for which we need to compute the partial derivatives is $\frac{\partial B}{\partial F}$. By expanding the partial derivatives and using the definition and derivatives of $C$ (Eqs. 3.44 and 3.45) we obtain:

$$
\frac{\partial B_{i j}}{\partial F_{p q}}=\left(\frac{\partial \operatorname{trace}(L)}{\partial F_{p q}}-\frac{\partial\left(n_{r} L_{r s} n_{s}\right)}{\partial F_{p q}}\right) C_{i j}+\left(\operatorname{trace}(L)-n_{r} L_{r s} n_{s}\right) \frac{\partial C_{i j}}{\partial F_{p q}}+\frac{\partial C_{i j}}{\partial F_{p q}}
$$

In the incremental formulation $L=I-F_{\text {old }} F^{-}$hence by using Eq. 3.51 we obtain.

$$
\frac{\partial L_{i j}}{\partial F_{p q}}=-F_{o l d, i k} F_{k p}^{-} F_{q j}^{-} .
$$

By noting the derivative of the trace of tensor with respect to its components is $\delta_{i j}$ and using Eq. 3.54 the identity matrix, we can compute the derivate of velocity gradient with respect to the deformation gradient as:

$$
\frac{\partial \operatorname{trace}(L)}{\partial F_{p q}}=\frac{\partial \operatorname{trace}(L)}{\partial L_{i j}} \frac{\partial L_{i j}}{\partial F_{p} q}=-\delta_{i j} F_{o l d, i k} F_{k p}^{-} F_{q j}^{-}
$$

Now we are left with computing the derivative of the second term inside the parentheses in Eq. 3.53. By recalling that $n_{i}=R_{i j} N_{j}$ and substituting we obtain:

$$
\frac{\partial\left(n_{r} L_{r s} n_{s}\right)}{\partial F_{p q}}=\frac{\partial R_{r i}}{\partial F_{p q}} N_{i} L_{r s} R_{s j} N_{j}+R_{r i} \frac{\partial L_{r s}}{\partial F_{p q}} R_{s j} N_{j}+R_{r i} N_{i} L_{r s} R_{s j} \frac{\partial R_{s j}}{\partial F_{p q}} N_{j}
$$

where the partial derivatives on the right hand side can be computed using Eqs. 3.46 and 3.54 .

To complete the Jacobian definition, we need to compute the derivative of $\frac{\partial \llbracket \Delta u \rrbracket^{I}}{\partial \llbracket u \rrbracket}$ present in Equation 3.41. By using Eq. 3.7 we can write:

$$
\frac{\partial \llbracket \Delta u \rrbracket_{i}^{I}}{\partial \llbracket u \rrbracket_{j}}=\frac{\partial\left(\Delta Q_{i k} \llbracket u \rrbracket_{k}+Q_{i k} \llbracket \Delta u \rrbracket_{k}\right)}{\partial \llbracket u \rrbracket_{j}}=\Delta Q_{i k} \delta_{k j}+Q_{i k} \delta_{k j}=\Delta Q_{i j}+Q_{i j}
$$

where $\Delta Q=Q_{0} \Delta R^{T}$. The complete Jacobian can now be computed by assembling all the derivates that has been identified in this section. 


\subsection{Validation}

Validation of the objective traction rate was performed by using a simple linear elastic traction separation law:

$$
\Delta T_{i}=K_{i j} \llbracket \Delta u \rrbracket_{j}^{I}
$$

where $K$ is the interface stiffness matrix and is defined as:

$$
K=\left[\begin{array}{l}
K_{N}, 0,0 \\
0, K_{S}, 0 \\
0,0, K_{S}
\end{array}\right]
$$

with $K_{N}=1 \cdot 10^{7 \mathrm{MPa}} / \mathrm{mm}$ and $K_{S}=1 \cdot 10^{7} \mathrm{MPa} / \mathrm{mm}$. The solid elements have been modeled using isotropic elasticity with a Young's modulus of $E=1 \cdot 10^{4} \mathrm{MPa}$ and a Poisson's ratio of $\nu=0.3$. The values of the interface stiffness and bulk material properties have been selected to allow deformations mainly in the bulk material.

\subsubsection{Objective traction rate validation}

To validate the objective traction rate formulation we compare two cases: i) one with a cohesive interface, and ii) one without. If the traction objective rate is correct introducing a very stiff interface should not change the simulation results. Furthermore to check both large rotations and large area changes, the simulation includes an initial axial strain up to an axial strain of 1 in the loading direction $(z)$ and then a rigid rotation of $90^{\circ}$ around the $y$ axis (see Figure 3.2). We compare both the first Piola-Kirchoff traction, $T$ and the Cauchy's traction $t$. Figures 3.3 and 3.4 compares the first Piola-Kirchoof traction and Cauchy's traction for the two cases described above. The results of the two simulations are identical thus confirming the correct implementation of the model and the validity of Eq. 3.1.

\subsubsection{Cohesive zone model Jacobian validation}

To evaluate the accuracy of the analytical Jacobian we utilized a classic patch test. We used the same material and interface model described in the previous section with similar loading conditions (stretch and subsequent rotation). The patch test mesh is depicted in Figure 3.5 To ensure the correctness of the implemented analytic Jacobian we compared the converge rate for two simulations using: i) the implemented analytic Jacobian, and ii) the Jacobian computed using finite differences. If for a patch test the same convergence rate are observed for both cases then the analytic Jacobian is correct. Comparison of the number of nonlinear iterations required to achieve convergence using both kinds of Jacobians are presented in Table 3.1. The analytic Jacobian requires the same number of nonlinear iterations, therefore we conclude that the analytic Jacobian has been correctly implemented. 


\section{Time: 0}
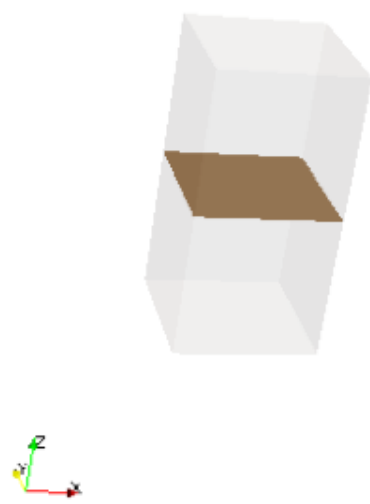

\section{Time: 1.5}

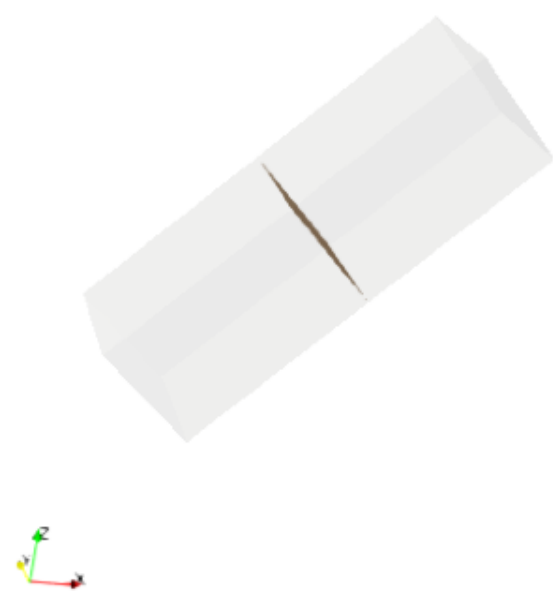

Time: 1

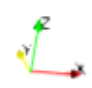

Time: 2
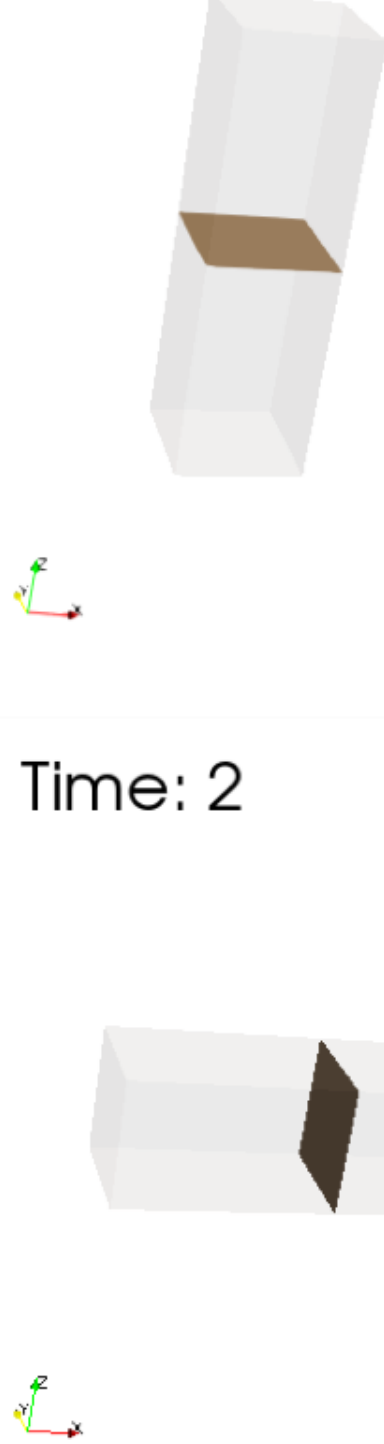

Figure 3.2: Rendering of the simulation without the CZM interface at different point in time. Axial stretching starts at time $=0$ and ends a time $=1$. The $90^{\circ}$ rigid body rotation around the $y$ axis starts at time $=1$ and ends a time $=2$. The highlighted surface shows where the traction are computed. 


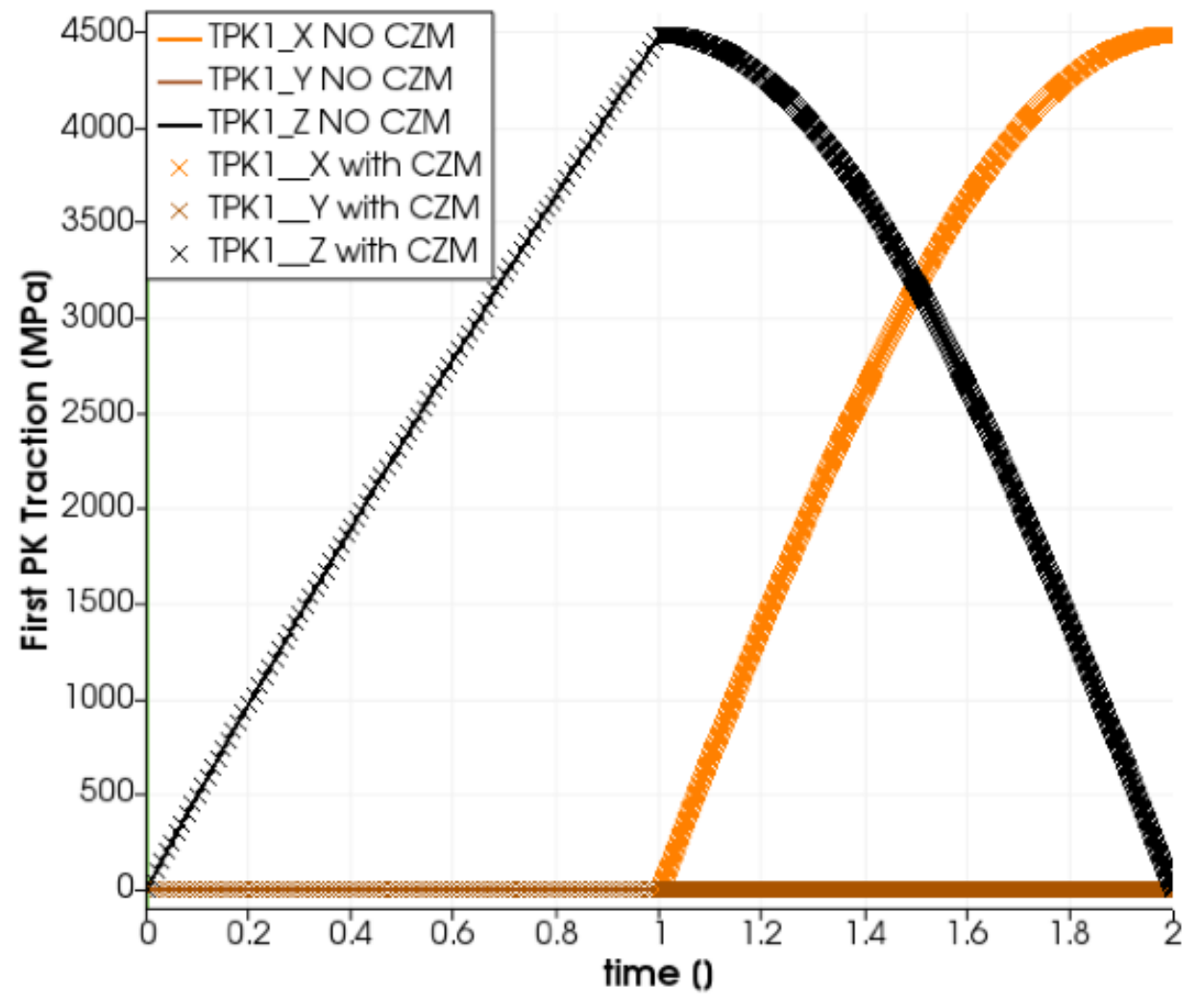

Figure 3.3: Comparison of the First Piola-Kirchoff traction for the two simulations with and without cohesive zone linear elastic model. Loading conditions are described in Figure 3.2. 


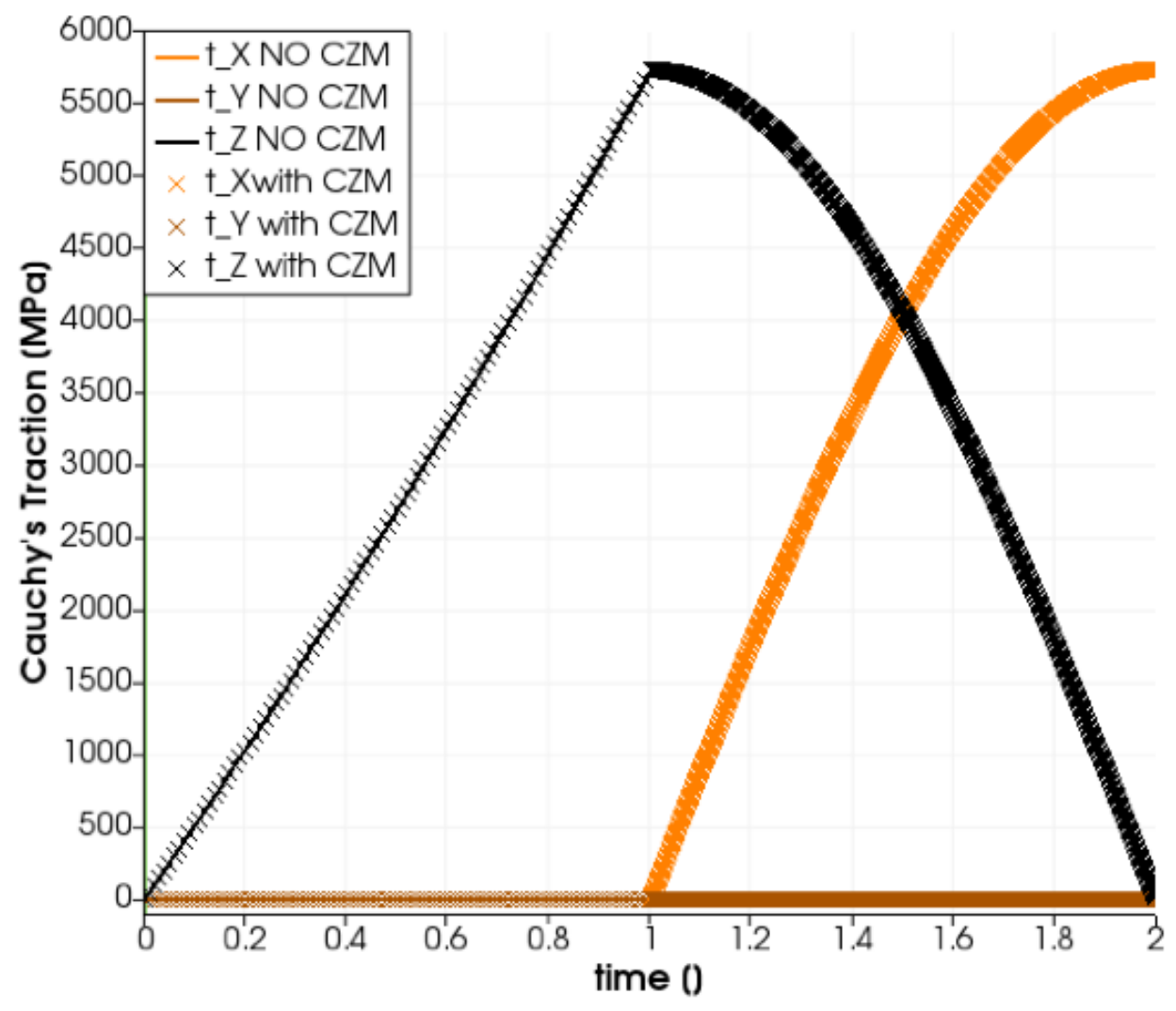

Figure 3.4: Comparison of the Cauchy traction for the two simulations with and without cohesive zone linear elastic model. Loading conditions are described in 3.2. 

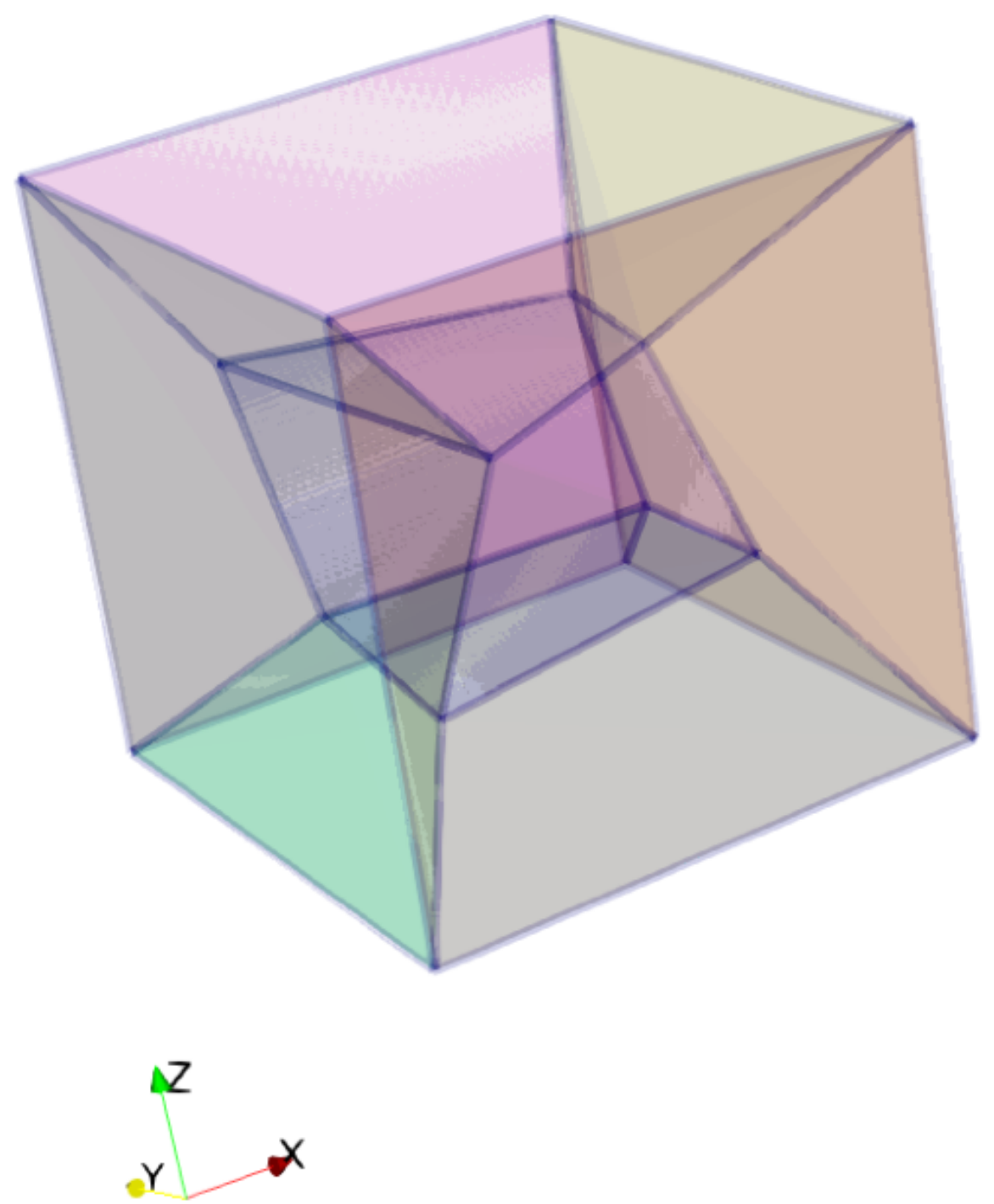

Figure 3.5: Render of the patch test mesh used to check the correctness of the implemented analytic Jacobian. Cohesive interfaces have been inserted between each and every element. 
Identify the influence of microstructure on mesoscale creep and fatigue damage

September 2020

\begin{tabular}{ccc}
\hline step & FDJ iterations & AJ iterations \\
\hline 1 & 3 & 3 \\
2 & 3 & 3 \\
3 & 3 & 3 \\
4 & 3 & 3 \\
5 & 4 & 4 \\
6 & 4 & 4 \\
7 & 4 & 4 \\
8 & 4 & 4 \\
\hline
\end{tabular}

Table 3.1: Comparison of number of nonlinear iteration required to achieve convergence when using: i) the finite difference calculated Jacobian (column FDJ), and ii) the analytic Jacobian (column AJ). 



\section{Constitutive models}

\subsection{The prior austenite grain model}

Creep cavity nucleation and growth predominantly occurs along prior austenite grain (PAG) boundaries in Grade 91. Therefore the crystal plasticity constitutive model ignores Grade 91 sub-grain structure. The framework used to model the deformation of the PAG is NEML [18]. NEML is a framework developed at Argonne National Laboratory and is compatible with MOOSE [11].

Following the example of Nassif et al. [3] our model uses isotropic elasticity to describe the elastic behavior of the grain bulk, a crystal plasticity based model to incorporate deformation caused by dislocation glide, and an isotropic plasticity-based model to incorporate diffusion creep. References [3, 4] describe this model in detail. This chapter summarizes key features. Both the crystal plasticity model and the isotropic plasticity model contribute to the symmetric part of the total plastic velocity gradient $D_{p}$ as:

$$
D_{p}=D_{\text {diff }}+D_{c p}
$$

The following equations describe the dislocation contribution to the deformation rate:

$$
\begin{aligned}
D_{c p} & =\operatorname{sym}\left(L_{c p}\right) \\
L_{c p} & =\sum_{s=1}^{12} \dot{\gamma}^{s}\left(m^{s} \otimes n^{s}\right) \\
\dot{\gamma}^{s} & =\dot{\gamma}_{0}\left(\frac{\tau^{s}}{\tilde{\tau}}\right)^{n} \\
\tilde{\tau} & =\tau_{0}+\tau_{w} \\
\dot{\tau}_{w} & =\theta_{0}\left(1-\frac{\tau_{w}}{\tau_{s a t}}\right) \sum_{s=1}^{N_{s s}}\left|\dot{\gamma}^{s}\right|
\end{aligned}
$$

where $s$ is the slip system index, $n^{s}$ is a slip system unit normal, $m^{s}$ is a slip system unit direction vector, $\tau^{s}$ is the resolved shear stress, $\dot{\gamma}^{s}$ the slip system shear rate, $\dot{\tau}_{w}$ is the slip resistance rate, and $N_{s s}$ is the number of slip systems. For all the simulations in this work we will consider only the 12 slip systems belonging to the $\{111\}\langle\overline{1} 10\rangle$ family.

The contribution of the diffusional creep term to the plastic velocity gradient is:

$$
D_{\text {diff }}=A \sigma_{V M} \cdot s
$$

where $s$ is the deviatoric part of the Cauchy stress, $\sigma, \sigma_{V M}$ is the von Mises stress, and $A$ is the diffusion constant. All the base model parameters values are presented and described in Table 4.1 . 


\begin{tabular}{llll}
\hline symbol & description & value & units \\
\hline$E$ & Young's modulus & $150 \cdot 10^{3}$ & $\mathrm{MPa}$ \\
$\nu$ & Poisson's ratio & 0.285 & unitless \\
$n$ & Voce hardening exponent & 12 & unitless \\
$\tau_{0}$ & initial slip resistance & 40 & $\mathrm{MPa}$ \\
$\tau_{\text {sat }}$ & saturation slip resistance & 12 & $\mathrm{MPa}$ \\
$\theta_{0}$ & slip hardening constant & 66.67 & unitless \\
$\dot{\gamma}_{0}$ & prefactor & $9.55 \cdot 10^{-8}$ & unitless \\
$A$ & Diffusional creep constant & $1.2 e \cdot 10^{-9}$ & unitless \\
\hline
\end{tabular}

Table 4.1: Grain bulk material parameters.

\subsection{Grain boundary cavitation model}

The grain boundary cavitation model is an improvement from the one described by Nassif et al. [3]. This model was initially conceived by Sham and Needleman [19] and later extended by Van Der Giessen et al. [20] to higher triaxiality regimes. There are several improvements to the model versus the version described in [3]:

- The model described in this work uses a viscoelastic traction separation law, thus allowing to correctly account for the instantaneous response of the material to sudden load changes. This is true for both opening and sliding traction (see Eq. 4.10).

- The high triaxiality cavity growth branch used to incorporate the cavity coalescence behavior was neglected. As explained in [13] the equation describing coalescence are not always stable and return unphysical results for situation they were not conceived for.

- According to the continuous cavity nucleation concept, the cavity nucleation criterion was modified to be a one time check (see Eq. 4.9). Once the criterion has been satisfied once during the loading history, cavities can nucleate continuously under a positive opening traction 4.9 .

- To enforce physical constraints on the state variables the material model uses Lagrange multiplier[21].

- To prevent the insurgence of unphysical traction oscillations related to grain innerpenetration the model utilize a continuous, quadratic-penalty approach [21].

These improvements were developed as part of another project, but they are included in the model used in the sensitivity and uncertainty quantification studies described in Chapter 5 .

The continuous cavitation grain boundary model accounts for cavities growth and cavity nucleation. Cavity growth is described by the evolution of the cavity half radius, $a$. The cavity average area density, $N$, is geometrically related to the average cavity half spacing, $b$, by $N=\frac{1}{\sqrt{\pi b^{2}}}$ [3]. Figure 4.1 is a schematic depicting the physical meaning of cavity half spacing and cavity half radius variables. 


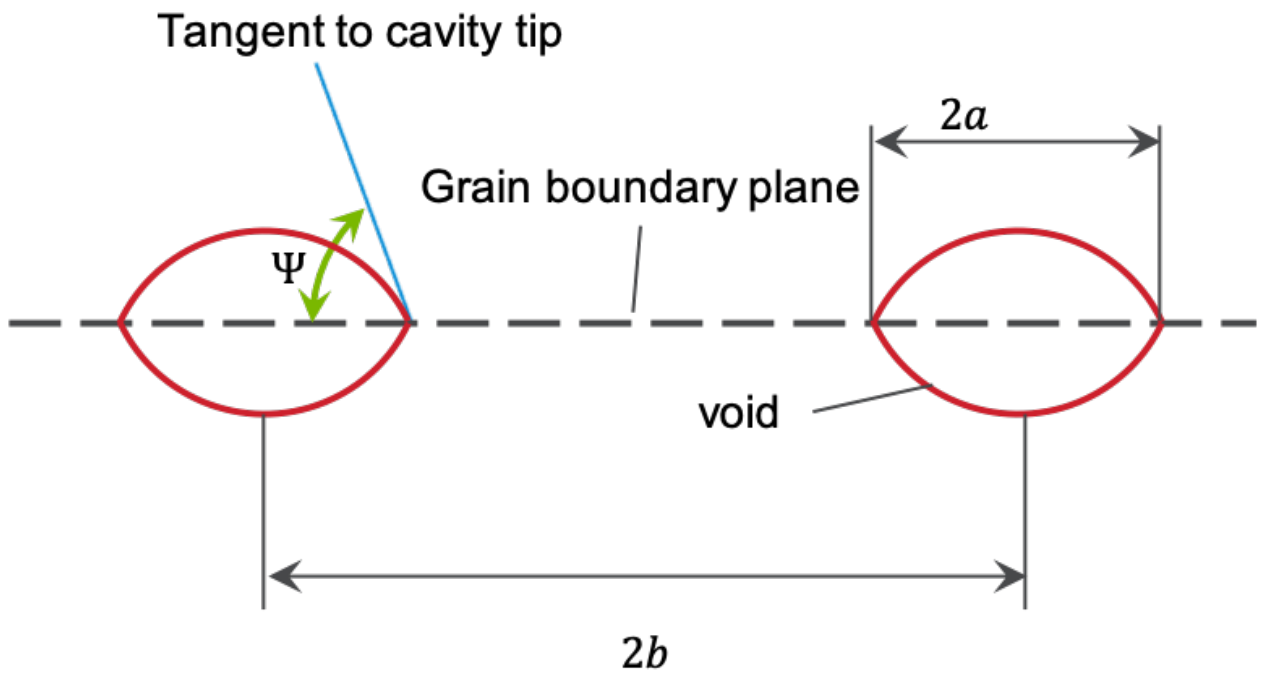

Figure 4.1: Schematic representing the physical meaning of the grain boundary cavitation model state variables. The variable $a$ represents the average cavity half radius, and the variable $b$ represents the average cavity half spacing.

$$
\begin{aligned}
& \dot{a}=\frac{\dot{V}}{4 \pi h(\Psi) a^{2}} \\
& \dot{b}=\left\{\begin{array}{c}
-\pi b^{3} F_{N}\left(\frac{\left\langle T_{N}\right\rangle}{\Sigma_{0}}\right)^{\gamma} \dot{\varepsilon}_{e q}^{C} \quad \text { if } \quad\left(\frac{\left\langle T_{N}\right\rangle}{\Sigma_{0}}\right)^{\beta} \int_{0}^{T}\left|\dot{\varepsilon}_{e q}^{C}\right| d t \geq \frac{N_{I}}{F_{N}} \text { once } \\
0 \text { otherwise }
\end{array}\right. \\
& \dot{T}_{N}=\left(\llbracket \dot{u} \rrbracket_{N}+\frac{\dot{V}\left(T_{N}\right)}{\pi b^{2}}\right) C_{N} \\
& \dot{T}_{S_{1}}=\left(\llbracket \dot{u} \rrbracket_{S_{1}}+\frac{T_{S_{1}}}{\eta_{G B} f_{S}}\right) C_{S} \\
& \dot{T}_{S_{2}}=\left(\llbracket \dot{u} \rrbracket_{S_{2}}+\frac{T_{S_{2}}}{\eta_{G B} f_{S}}\right) C_{S}
\end{aligned}
$$

The cavity growth equations are

$$
\begin{aligned}
& \dot{V}=\dot{V}^{D}+\dot{V}^{\text {triax }} \\
& \dot{V}^{D}=8 \pi D \frac{T_{N}}{q(f)}
\end{aligned}
$$




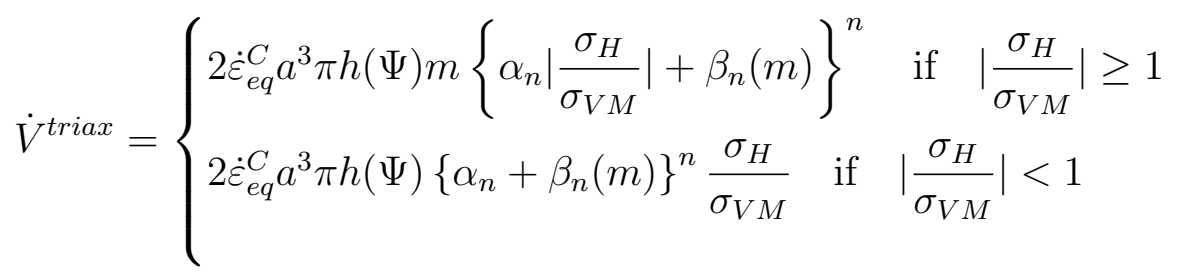

with

$$
\begin{aligned}
& f=\max \left(\frac{a^{2}}{(a+1.5 L)^{2}}, \frac{a^{2}}{b^{2}}\right), \quad L=\left(\frac{D \sigma_{V M}}{\dot{\varepsilon}_{e q}^{C}}\right)^{\frac{1}{3}} \\
& q(f)=2 \log \left(\frac{1}{f}\right)-(1-f)(3-f) \\
& h(\Psi)=\left(\frac{1}{1-\cos (\Psi)}-\frac{\cos (\Psi)}{2}\right) \frac{1}{\sin (\Psi)} \\
& m=\operatorname{sign}\left(\sigma_{H}\right) \\
& \beta(m)=\frac{(n-1)[n+g(m)]}{n^{2}} \\
& g(m)=\left\{\begin{array}{l}
\log (3)-\frac{2}{3} \quad \text { if } \quad m=1 \\
\frac{2 \pi}{9 \sqrt{3}} \quad \text { if } \quad m=-1 \\
0 \quad \text { if } \quad m=0
\end{array}\right. \\
& \alpha_{n}=\frac{3}{2 n} \quad \begin{array}{l}
\text { (f } \\
g(m)
\end{array}
\end{aligned}
$$

Detailed implementation of the model including: i) the method used to enforce physical constraint on cavitation state variables, and ii) the methodology used to impose a smooth inner-penetration penalty are described in [21]. Table 4.2 describes all the model parameters and their calibrated values used as the starting point for the uncertainty quantification studies in the next chapter. 


\begin{tabular}{llll}
\hline symbol & description & value & units \\
\hline$\beta$ & traction nucleation exponent & 2 & unitless \\
$n_{G B}$ & creep rate exponent & 5 & unitless \\
$a_{0}$ & initial cavities half radius & $5 \cdot 10^{-5}$ & $\mathrm{~mm}^{2}$ \\
$b_{0}$ & initial cavities half spacing & 0.06 & $\mathrm{~mm}^{2}$ \\
$D$ & grain boundary diffusion coefficient & $1 \cdot 10^{-15}$ & $\mathrm{~mm}^{3} / \mathrm{MPa} \cdot \mathrm{h}$ \\
$\Psi$ & cavity half tip angle & 75 & $\circ$ \\
$\Sigma_{0}$ & traction normalization parameter & 200 & $\mathrm{MPa}$ \\
$\frac{F_{N}}{N_{I}}$ & normalized nucleation rate constant & $2 \cdot 10^{4}$ & $1 / \mathrm{mm}^{2}$ \\
$\frac{N_{\max }}{N_{I}}$ & normalized maximum cavity density & $1 \cdot 10^{3}$ & $\mathrm{unitless}$ \\
$E_{G B}$ & interface Young modulus & $150 \cdot 10^{3}$ & $\mathrm{MPa}$ \\
$G_{G B}$ & interface in-plane Shear modulus & $58.63 \cdot 10^{3}$ & $\mathrm{MPa}$ \\
$\eta_{G B}$ & sliding viscosity & $1 \cdot 10^{6}$ & $\mathrm{MPa} \cdot \mathrm{h} / \mathrm{mm}$ \\
\hline
\end{tabular}

Table 4.2: Grain boundary cavitation material parameters. 



\section{Identification of microstructural parameters influencing creep rupture life}

The physically-motivated model describing the creep behavior of Grade 91 has two main components. The prior austenite grain (PAG) model and the grain boundary cavitation (GBC) model. Previous work calibrated the PAG and GBC models to fit the deterministic average of a set of Grade 91 experimental [3, 12, 22, 23]. However, a deterministic model cannot capture the experimentally observed variability in creep-rupture life. Figure 5.1 depicts the experimentally observed distribution of Grade 91 creep rupture life at $600{ }^{\circ} \mathrm{C}$ for a nominal stress of $100 \mathrm{MPa}$. The black line shows the computed time to creep rupture using the calibrated parameters.

Both the PAG and GBC models include the simulated creep curve and rupture life. As both models are physically-based, their parameters aim to describe the evolution of microstructural deformation and failure mechanism of Grade 91. The PAG model incorporates two different mechanisms: i) point defect diffusion, and ii) dislocation creep. Point defects diffusion is related to the chemical potential, enhanced by temperature and driven by stress gradients. The accumulation and flux of point defect results in a measurable strain contribution. The PAG model incorporate point defect diffusion utilizing an isotropic linear diffusion model that generates a strain rate proportional to the deviatoric stress and a diffusion coefficient $A$. Dislocation creep is modeled utilizing a crystal plasticity (CP) approach [24] which uses a power-law to determine the amount of dislocation glide, and includes a slip resistance strengthening term to mimic the apparent slip resistance increase observed in experiments $[14,25]$.

The GBC incorporates three distinct mechanisms: i) cavity growth, ii) cavity nucleation, and iii) grain boundary sliding. These three mechanism are interlaced with each other through a set of coupled rate equations describing the evolution of void nucleation and cavitation. The GBC model assumes continuous nucleation and continuous growth of cavities generating from carbides present at the grain boundaries. The cavity nucleation rate is governed by two parameters: a prefactor $F_{N}$ and a traction normalization value $\Sigma_{0}$. The cavity nucleation rate is modeled via the variable $b$, which physically represents the half space between two adjacent cavities. The cavity growth process is mathematically expressed in terms of the average cavity half radius $a$, which is geometrically linked to the grain boundary opening via a geometric relationship. The only three calibrated parameters in this model are the grain boundary diffusivity $D_{G B}$, the equilibrium cavity shape angle $\Psi_{G B}$, and the cavity growth exponent $n_{G B}$. Besides the evolution parameters, the GBC model requires the initial number of cavities, $a_{0}$ and the initial cavity spacing $b_{0}$.

The calibrated parameters will be the baseline for the sensitivity analysis. More details about the PAG and the GBC models, including equations and parameters values, can be found in sections 4.1 and 4.2. Table 4.1 and 4.2 list all the parameters and they calibrated values for the PAG and GBC models, respectively.

\subsection{Sensitivity analysis}

Sensitivity analysis is a well known technique to identify the most sensitive parameters of a model with respect to a target scalar variable. A full sensitivity analysis requires exploring 


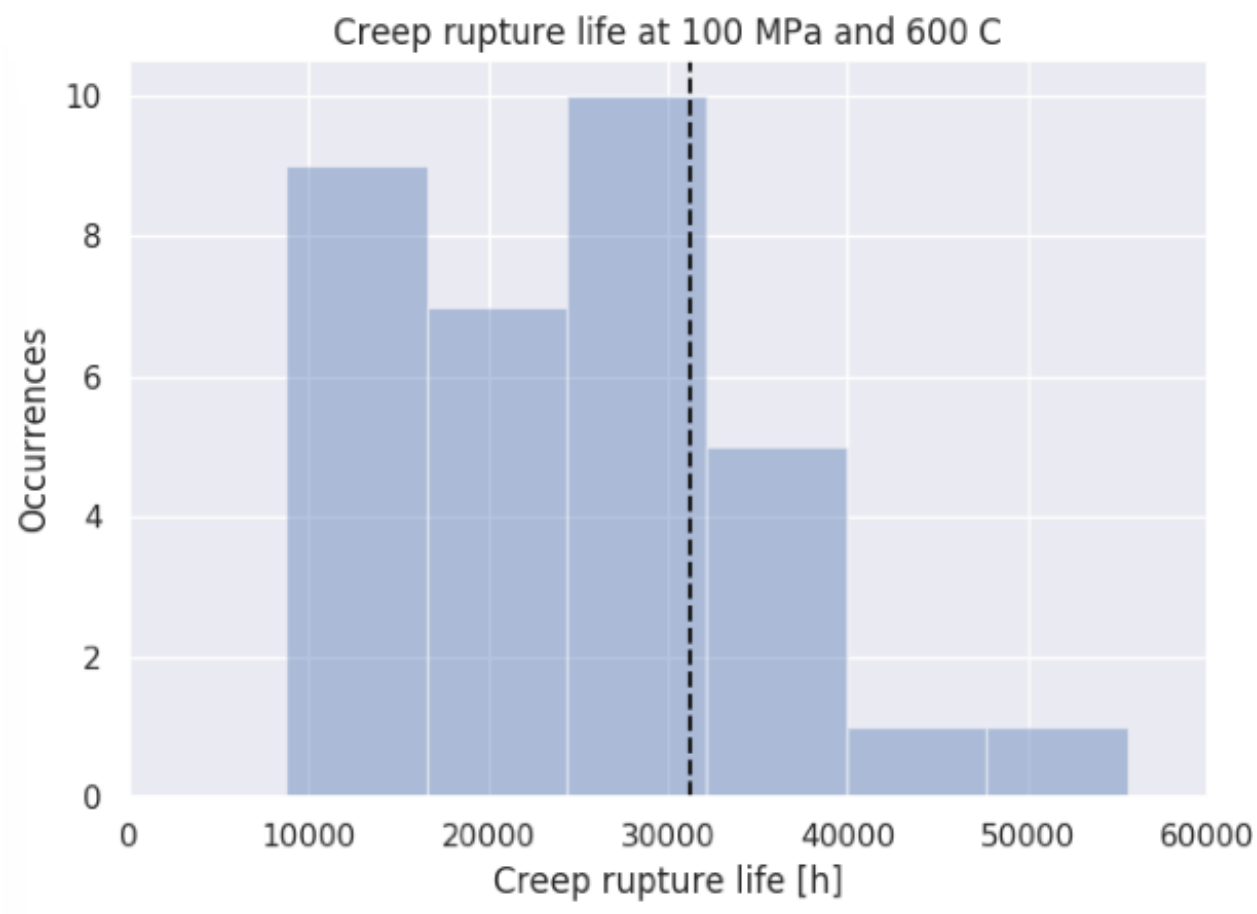

Figure 5.1: Experimentally observed distribution of Grade 91 creep rupture life at $600{ }^{\circ} \mathrm{C}$ for a nominal stress of $100 \mathrm{MPa}$. The black line represents simulation results

the full parameter space and is usually very expensive. Most of the times hundreds, if not thousands of simulations are required. The numberer of simulations required to fully explore the parameter space increases with the number of parameters. Crystal plasticity simulation are computationally expensive because the material requires solving a complicated nonlinear system at each integration point whose size linearly increases with the number of slip systems and state variables. Bandyopadhyay et al. [26] developed a methodology applying a genetic algorithm rather than a Monte Carlo approach to reduce the number of required simulations. They claim their approach reduces the number of required simulations between 100 and 1000 times. However, for a crystal plasticity model with eight parameters they still had to perform more than 7000 simulations. Therefore, performing a full sensitivity analysis on a crystal plasticity model with more than 20 parameters (like the model here) is practically unfeasible. Therefore, we decide to perform a one-at-a-time (OAT) sensitivity analysis using the deterministic, mean-property calibrated parameters as a starting point. The OAT analysis varies one model parameter at a time and observes the effect on the final output.

For our case the variable of interest is the creep-rupture time under uniaxial loading conditions. To mimic creep experimental loading conditions we used box-periodic boundary conditions. Box periodic BC simulates periodicity by using three symmetry planes and imposing an equal displacement on the remaining faces. Furthermore, to simulate uniaxial loading, a nominal traction boundary condition is imposed on one on the face corresponding to the loading direction. Figure 5.2 is a schematic illustration of the applied box-periodic BC. The applied nominal traction of $100 \mathrm{MPa}$ was selected to probe the two different deformation 


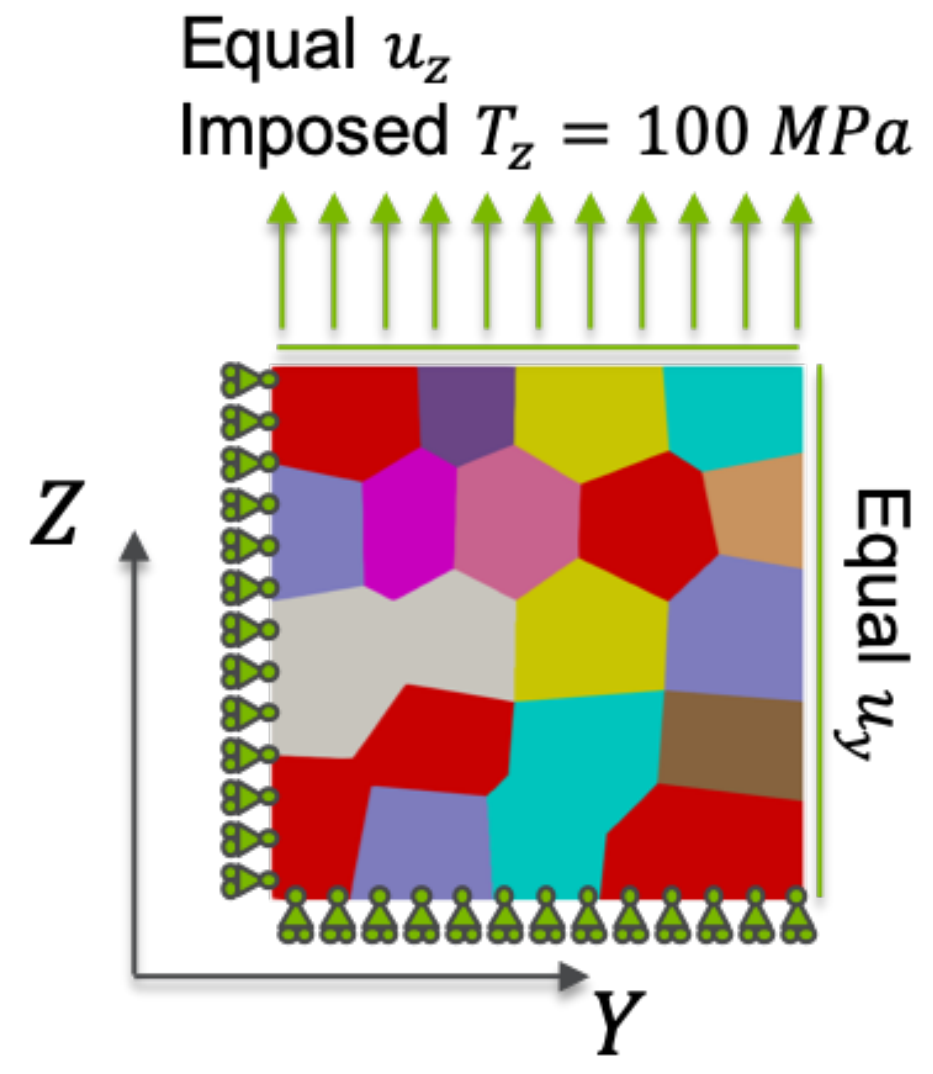

Figure 5.2: Schematic representing box-periodic boundary conditions for uniaxial creep loading. The colored block represents the RVE and each color represents a grain. $T_{Z}$ is the nominal traction and corresponds to a constant applied force.

regimes observed for Grade 91 [3, 27]. $100 \mathrm{MPa}$ is the watershed between the diffusional and dislocation bulk grain deformation mechanism. Figure 5.3 depicts simulation results for an applied nominal traction of $100 \mathrm{MPa}$.

During experiments, the rupture time can be easily measured as the time until the specimen breaks in half. However, including failure in stress controlled finite element simulations is challenging. When damage is widespread the structure will not be able to sustain the imposed load, and the finite element simulation will have difficulty converging, thus requiring small time steps, greatly increasing the cost of the simulations. Therefore we define a stopping criteria short of complete RVE failure. To examine the impact of this criteria on the sensitivity analysis, we consider three options:

1. the time to $1 \%$ interface strain (TT1) in the loading direction,

2. the time to $2 \%$ interface strain (TT2) in the loading direction,

3. the time to $3 \%$ interface strain (TT3) in the loading direction.

If the sensitivity analysis provides the same results for all of the specified failure metrics 
Identify the influence of microstructure on mesoscale creep and fatigue damage

September 2020
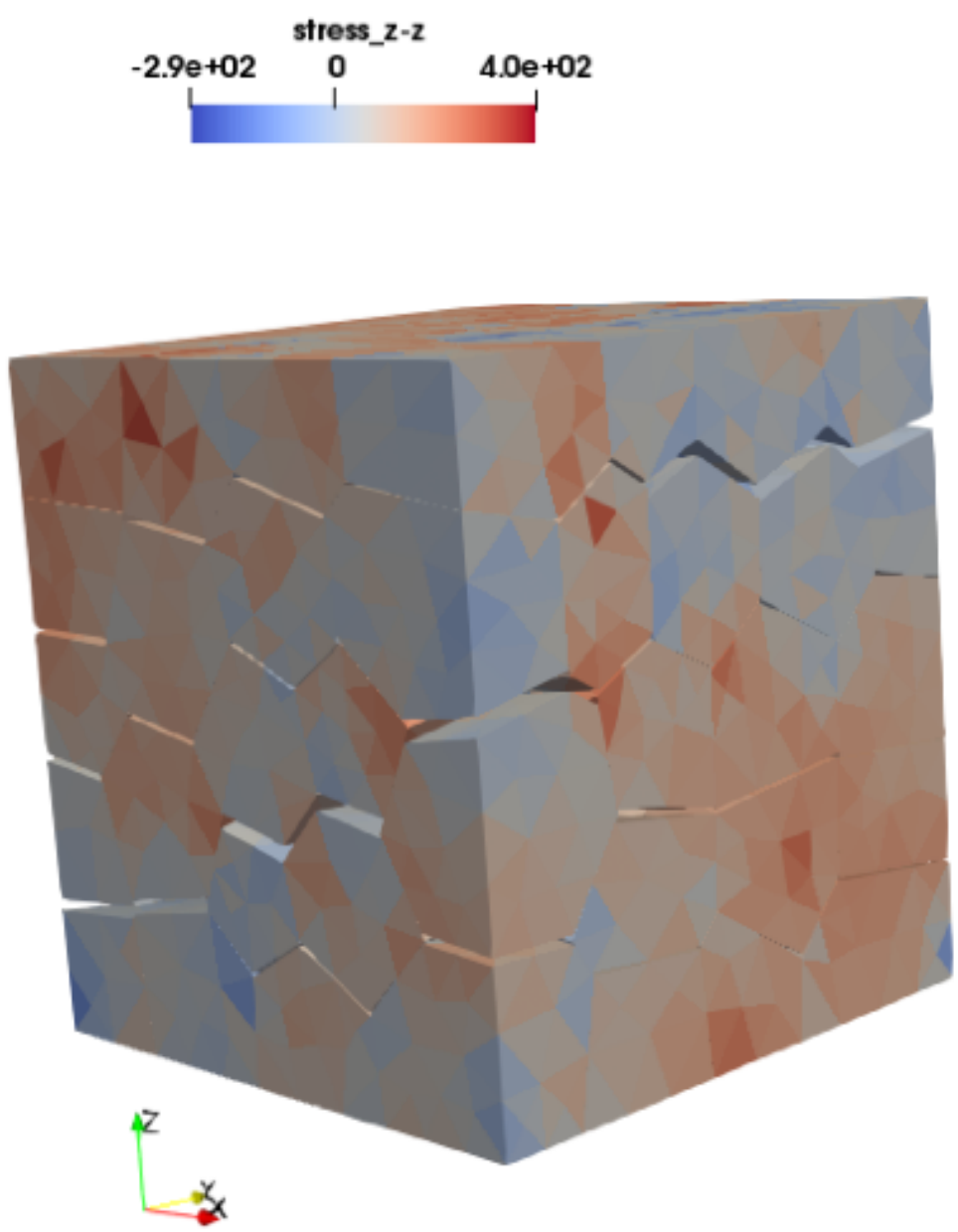

Figure 5.3: Rendering of simulation results for uniaxial creep and a nominal stress of $100 \mathrm{MPa}$. 
then it can be considered unbiased. The interface strain contribution is computed as:

$$
\begin{aligned}
\bar{\varepsilon}_{G B, i j} & =\frac{\int_{A} l_{G B, i j} d A}{V_{0}} \frac{A_{0}}{A} \\
l_{G B, i j} & =\frac{\llbracket u \rrbracket_{i} n_{j}+n_{i} \llbracket u \rrbracket_{j}}{2}
\end{aligned}
$$

where $A$ and $A_{0}$ are the deformed and undeformed grain boundary area, $V_{0}$ is the initial volume, $\llbracket u \rrbracket$ is the displacement jump, and $n$ is the interface normal in the deformed configuration.

The OAT analysis is performed by increasing each model parameter by a fix ratio, $R=$ $1 \cdot 10^{-6}$ and then computing the variation of the outcome as:

$$
S_{p}^{*}=\left|\frac{T T^{*}(p(1+R))-T T^{*}(p)}{p R}\right|
$$

where $T T^{*}$ is any of the failure metrics. A few parameters of the PAG and GBC models are related to each other. Specifically, the interface Young's and shear modulus, $E_{G B}$ and $G_{G B}$, are always assumed to be equal to the bulk material. Furthermore, the grain boundary diffusivity, $D_{G B}$ and the grain boundary viscosity, $\eta_{G B}$, are assumed to be related by [22, 28]:

$$
\eta_{G B}=\frac{C}{D_{G B}}
$$

where $C=1 \cdot 10^{-9} \mathrm{~mm}^{2}$ is the proportionality constant computed using the calibrated deterministic values of $D_{G B}$ and $\eta_{G B}$.

Results of the OAT sensitivity analysis are reported in Fig. 5.4. The first noticeable feature is the independence of the sensitivity from the selected failure metric. This implies that any of the three metric is equally good to identify the most relevant model parameters. In what follow we will use the time to $1 \%$ interface strain, TT1, as the failure metric. Furthermore, the results also show that the most sensitive parameter is the grain boundary diffusivity, $D_{G B}$. This result is not surprising and confirms that diffusional cavity growth is the dominant failure mechanism. This also implies that grain boundary viscosity might play a role because the viscosity is related to the diffusivity by Eq. 5.5. The second most influential parameter among the grain boundary model parameters is the initial cavity half radius, $a_{0}$, which is related to the size of second phase particle distribution at grain boundaries. The The most sensitive parameter for the grain bulk is the point defect diffusivity constant $A$. The grain bulk diffusivity is responsible for contributing to the total inelastic strain rate (Eq. 4.7), and therefore contributes to the triaxial cavity growth rate (Eq. 4.15). Conversely parameter related to dislocation glide, like, $\dot{\gamma}_{0}, \tau_{0}, \tau_{\text {sat }}$, and $\theta$, show a lesser effect on the creep failure time. Their lower effect might be related to the low applied stress $(100 \mathrm{MPa})$. Furthermore the, small effect of the dislocation creep parameters implies that diffusional creep is the dominant deformation mechanisms at this regime. A more comprehensive sensitivity analysis should be performed to fully investigate the effect of all the parameters on the creep behavior of Grade 91. A viable and simple approach would be to repeat the above analysis for different nominal traction levels, to identify at least the most sensitive parameter at each level. 


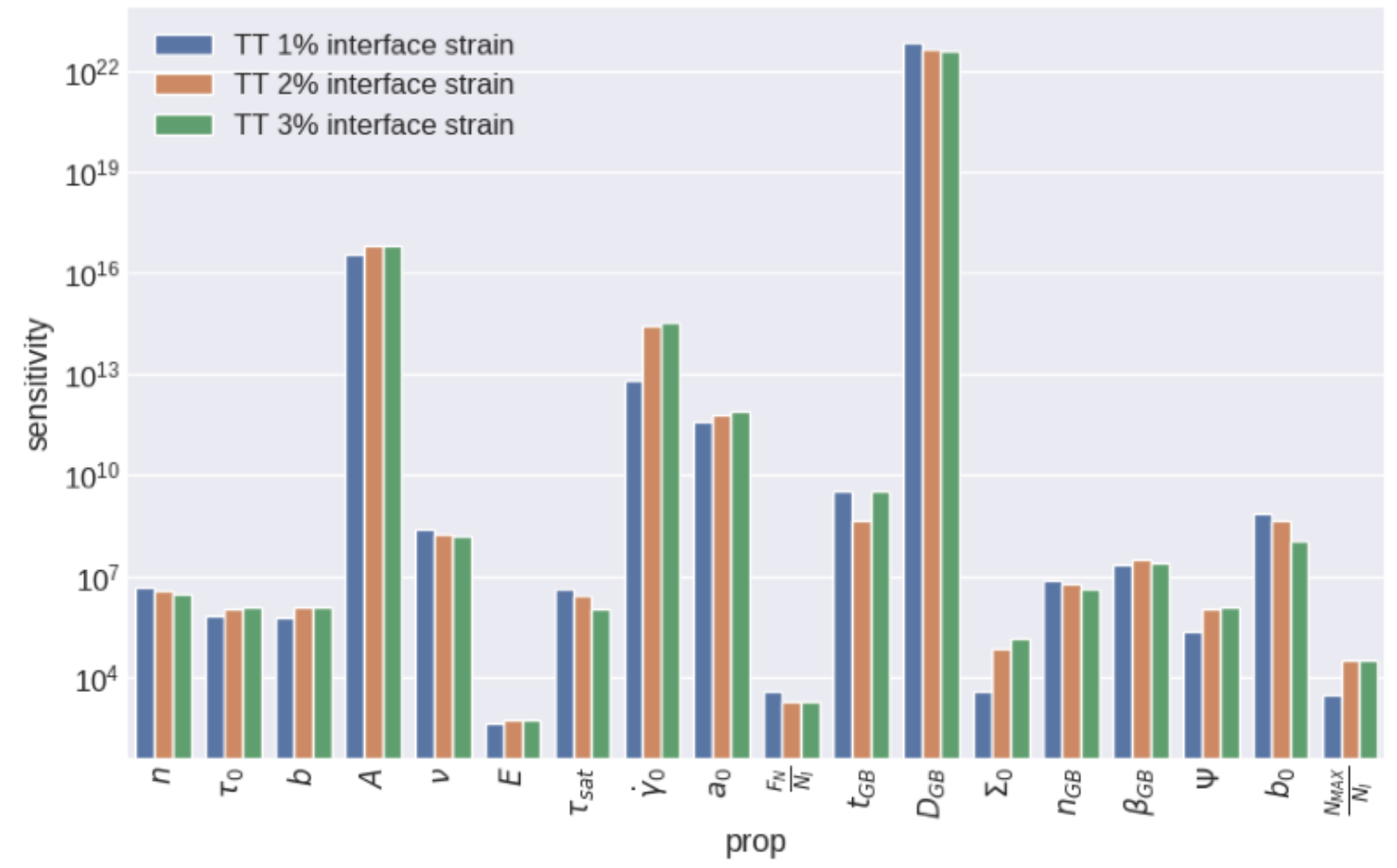

Figure 5.4: Sensitivity of different failure metrics with respect to the model parameters when performing a one-at-a-time sensitivity analysis. The failure metrics are: i) time to $1 \%$ interface strain (TT1), ii) time to $2 \%$ interface strain (TT2), and iii) time to $3 \%$ interface strain (TT3). Sensitivity is plotted in log space. 


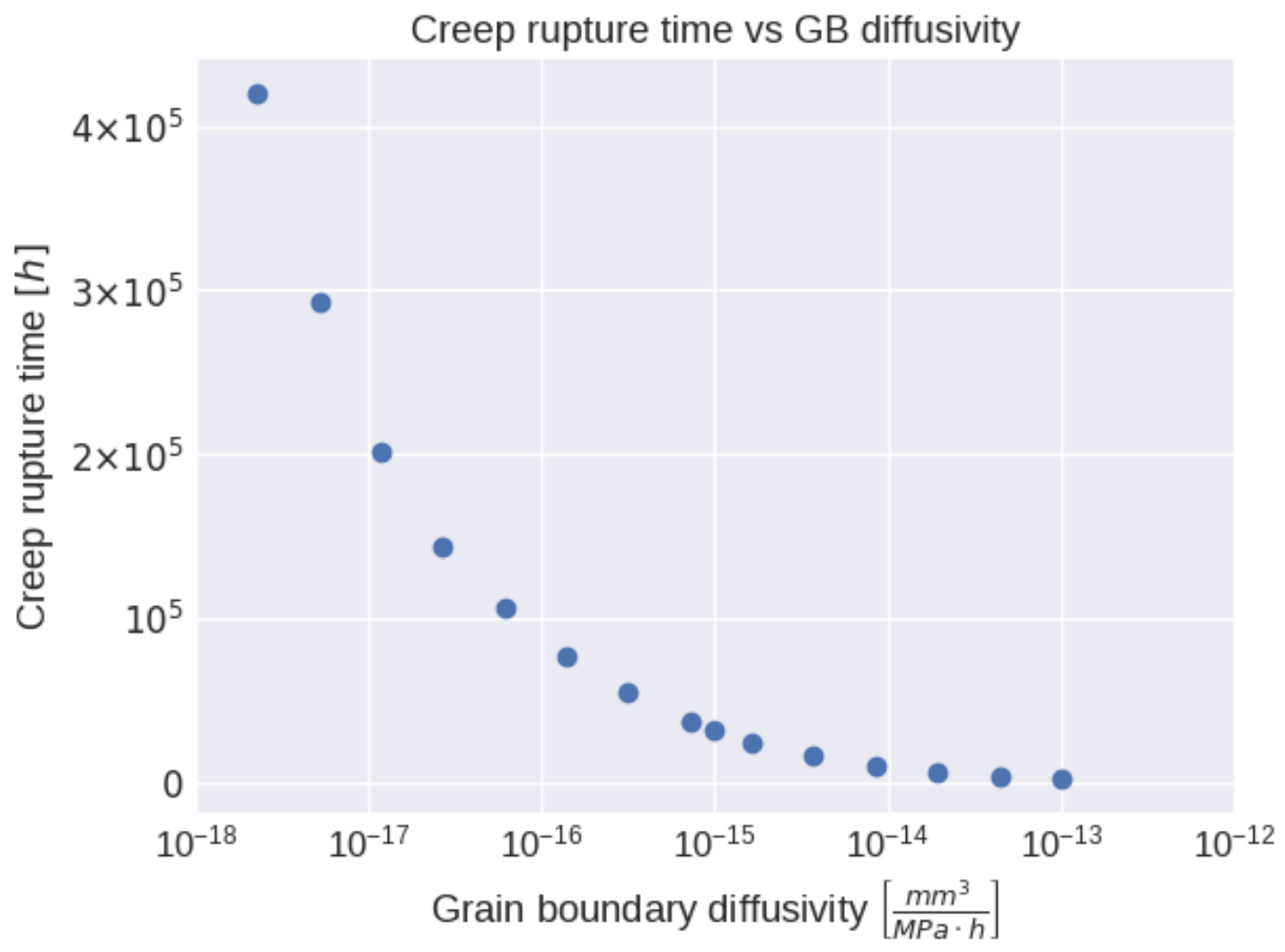

Figure 5.5: Computed time to failure, using the time to $1 \%$ interface strain metric, as function of the grain boundary diffusivity.

\subsection{Identifying parameters distributions}

As a first approximation we assumed that the grain boundary diffusivity is the only parameter affecting the computed creep rupture life. While this is a crude approximation, it can be justified by noticing that the grain boundary diffusivity exhibits a sensitivity six orders of magnitude higher than all the other parameters. Under this assumption, the computed creep-rupture life is a univariate distribution of the grain boundary diffusivity. Therefore the grain boundary diffusivity distribution can be computed by identifying the relationship between the grain boundary diffusivity and the computed creep rupture life, i.e. TT1 $\left(D_{G B}\right)$, and then constructing the $D_{G B}$ distribution starting from the experimental observations. To identify such relationship we performed a set of simulations changing the value of the grain boundary diffusivity. Figure 5.5 depicts the computed time to failure as function of the grain boundary diffusivity.

The grain boundary diffusivity distribution can be recovered by using piecewise interpolation to calculate the diffusivity given the observed experimental time to failure. Figure 5.6 depicts the discrete and continuous grain boundary diffusivity distribution. The original, deterministic calibrated parameter value is very close to the most frequent computed value, thus implying that the deterministic model correctly captures the mean behavior of Grade 


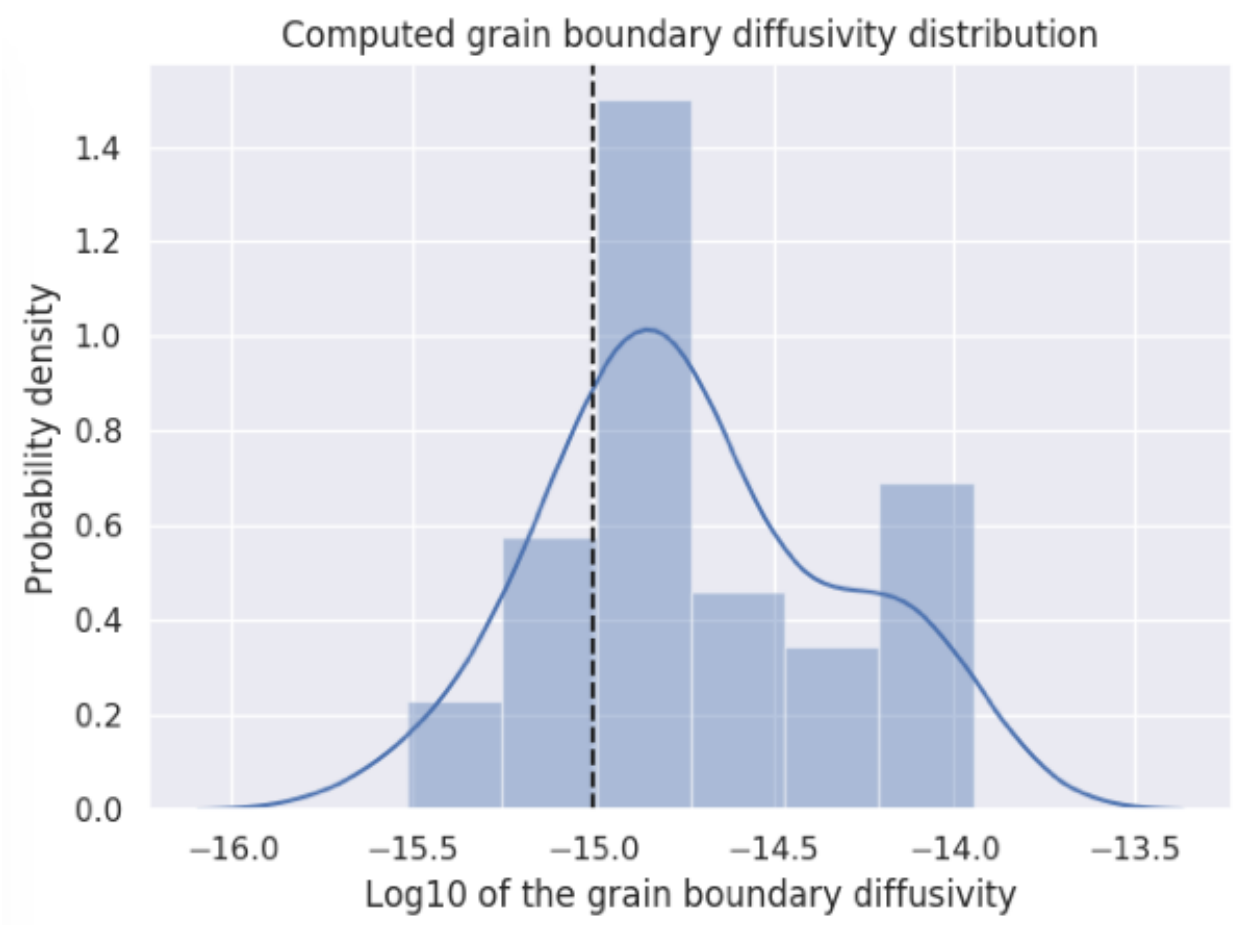

Figure 5.6: The numerically computed grain boundary diffusivity distribution. Vertical line represent the calibrated $D_{G} B$ value. The blue line represents a smooth distribution calculated using the kernel density estimation.

91. The width of the diffusivity distribution is approximately three orders of magnitude, which reasonably matches experimental measurements. McGarrity et al. [29] showed that the variation of diffusivity between low energy and high energy grain boundaries is approximately 10 orders of magnitude. Cao et al. [30] Showed that the excess free volume is linearly correlated to the grain boundary energy. Furthermore, it is reasonable to assume that the grain boundary diffusivity is linearly related to excess free volume, because a larger free volume would ease the diffusion process. Hence the diffusivity can be assumed to be correlated to the grain boundary energy. Ratanaphan et al. [31] computed the free surface energy of more than 400 grain boundaries, including very low energy and random grain boundaries. Normalizing their results shows that variability of the grain boundary energy for random grain boundaries is about $30 \%$ (see Figure 5.7). Hence, by assuming a log-linear correlation between the grain boundary diffusivity and the grain boundary energy, a $30 \%$ variability in energy can explain the 3 orders of magnitude variability on the grain boundary diffusivity .

The other parameters exhibiting high sensitivity should also be incorporated in the model for a full sensitivity analysis. Also our analysis does not account for correlations between different parameters, except for the correlation between diffusivity and sliding viscosity discussed in Section 5.1. 


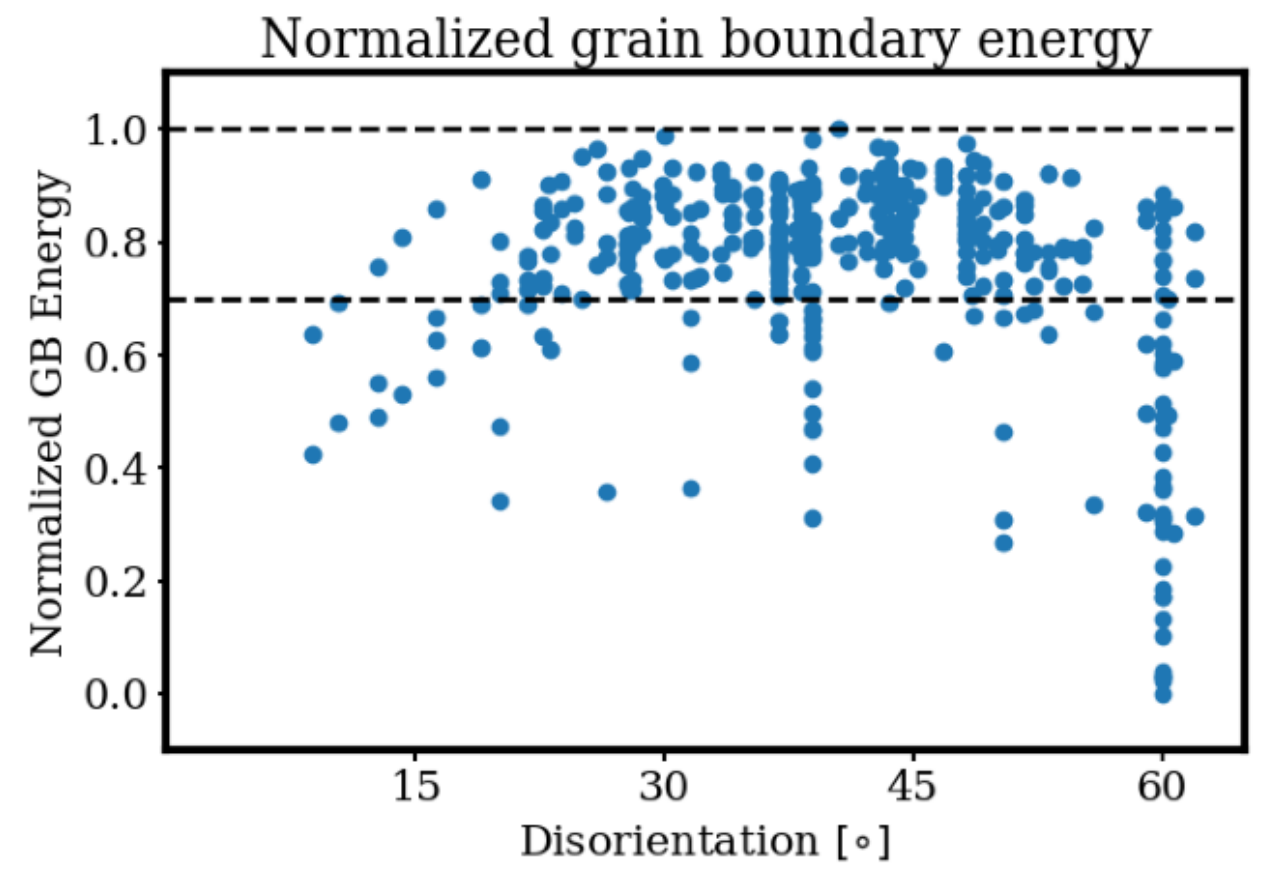

Figure 5.7: Normalized grain boundary energy. The dashed line shows the variability of the energy for random grain boundaries. Data from [31] 



\section{Conclusions and future work}

This report includes both improvements to MOOSE framework and a preliminary microstructural model capturing the observed scatter of the creep rupture life of Grade 91.

Improvements to the MOOSE framework include:

- The addition of a large-deformation kernel to the tensor mechanics module allowing MOOSE to use a total Lagrangian formulation. The new kernel also include the analytically correct Jacobian.

- A new scalar kernel system imposing average stress and strain for arbitrarily-shaped unit cells while maintaining the Hill-Mandel condition.

- A new mesh generator that automatically detects periodic boundaries for arbitrarilyshaped unit cells. The mesh generator was designed to allow users to directly utilize periodic Voronoi tessellation generated by Neper without utilizing any kind of external preprocessing tool.

- A new large-deformation cohesive zone model accounting for large area changes and large rotations of the interface. The introduction of a large deformation cohesive zone system was necessary to account for the large area changes observed during creep tests. This system includes both a new material and a new kernel. The new system also also includes the analytically correct Jacobian.

All the above improvements together will allow MOOSE users to perform true periodic crystal plasticity simulations without the need of meshing tools or preprocessing scripts, while utilizing a consistent stress measure. Pull requests to merge all the above improvements into MOOSE are in preparation and could be used in future modeling efforts.

The microstructural model for creep in Grade 91 was used to identify key microstructural parameters controlling creep failure:

- A one-at-a-time sensitivity analysis was performed to identify the parameters most influencing the computed creep-rupture life of Grade 91 at a constant nominal stress of $100 \mathrm{MPa}$. Results of the sensitivity analysis showed that the most influential model parameter is the grain boundary diffusivity.

- By using the sensitivity analysis results we showed that the experimentally observed creep-rupture life scatter can be accounted for utilizing a univariate statistical model only including the distribution of the grain boundary diffusivity.

- The obtained grain boundary diffusivity distribution was reasonable because its computed variability is comparable to the variability of the grain boundary energies.

While the proposed probabilistic model is able to capture the statistical distribution for creep-rupture life of Grade 91 steel further validation is need to demonstrate its applicability to a wider range of applied stresses.

Furthermore it would be of scientific and engineering interest to include the variability of other parameters to better capture the experimentally observed deformation and failure variability. For instance recent experiments performed at the Advanced Photon Source show 
that not all the favorably oriented grain boundaries will experience cavitation. This is in contrast with the baseline results of the utilized physically-based micro-mechanical model [4]. The most probable cause of this behavior is the homogeneity of grain boundary properties in the model. There is experimental evidence [32,33] and numerical studies [12,34] showing that accounting for the grain boundary character greatly increase the predictive capability of grain boundary models. Bulatov et al. [35] proposed a methodology computing the grain boundary energy given the grain boundary character, Ratanaphan et al. [31] found correlations between the grain boundary energy and grain boundary properties. Incorporating the grain boundary character in the micro-mechanical model will remove the assumption that all grain boundaries are equal and will incorporate the inherent grain boundary properties variability observed in experiments.

In addition to this improvement to the model physics, better numerical methods will be needed for UQ analysis through the CPFEM model. Chapter 5 cites [26], which promises a significant reduction in computational cost versus a Monte Carlo method. This technique could be explored. Additionally, improvements to the model numerics to reduce computation time will directly translate to reducing the time required for UQ.

Finally, this general approach could be extended to creep-fatigue simulations. The introduction notes that the program focused in FY2020 on creep loading, but future work will extend to creep-fatigue. Here, numerical expense will be critical as cyclic loading requires large numbers of time steps simply to represent the load reversals. The methods described in the previous paragraph could be helpful. Additionally, cyclic extrapolation schemes could be developed to skip entire load cycles without significantly compromising the model's accuracy. 


\section{Acknowledgements}

The research was sponsored by the U.S. Department of Energy, under Contract No. DEAC0206CH11357 with Argonne National Laboratory, managed and operated by UChicago Argonne LLC. Programmatic direction was provided by the Nuclear Energy Advanced Modeling \& Simulation Program of the Department of Nuclear Energy. The authors grateful acknowledge the support and direction of Ben Spencer, at Idaho National Laboratory, National Technical Director Chris Stanek, at Los Alamos National Laboratory, and Federal Manager Dave Henderson. 



\section{Bibliography}

[1] R. W. Swindeman, M. L. Santella, P. J. Maziasz, B. W. Roberts, and K. Coleman. Issues in replacing Cr-Mo steels and stainless steels with $9 \mathrm{Cr}-1 \mathrm{Mo}-\mathrm{V}$ steel. International Journal of Pressure Vessels and Piping, 81(6):507-512, 2004. ISSN 03080161. doi: 10.1016/j.ijpvp.2003.12.009.

[2] J. R. DiStefano, V. K. Sikka, J. J. Blass, C. R. Brinkman, J. M. Corum, J. A. Horsk, R. L. Huddleston, J. F. King, R. W. McClung, and W. K. Sartory. Summary of Modified 9Cr-1Mo Steel Development Program 1975-1985. Technical report, Oak Ridge National Laboratory ORNL-6303, 1986.

[3] Omar Nassif, Timothy J. Truster, Ran Ma, Kristine B. Cochran, David M. Parks, M. C. Messner, and T. L. Sham. Combined crystal plasticity and grain boundary modeling of creep in ferritic-martensitic steels: I. Theory and implementation. Modelling and Simulation in Materials Science and Engineering, 27(7), 2019. ISSN 1361651X. doi: 10.1088/1361-651X/ab359c.

[4] M. C. Messner, Omar Nassif, Ran Ma, Timothy J. Truster, Kristine Cochran, David Parks, and T.-L. Sham. Combined crystal plasticity and grain boundary modeling of creep in ferritic-martensitic steels: II. The effect of stress and temperature on engineering and microstructural properties. Modelling and Simulation in Materials Science and Engineering, 27:075010, 2019.

[5] Andrea Rovinelli, Henry Proudhon, Ricardo A Lebensohn, and Michael D Sangid. Assessing the reliability of fast fourier transform-based crystal plasticity simulations of a polycrystalline material near a crack tip. International Journal of Solids and Structures, 184:153-166, 2020.

[6] Romain Quey, PR Dawson, and Fabrice Barbe. Large-scale 3d random polycrystals for the finite element method: Generation, meshing and remeshing. Computer Methods in Applied Mechanics and Engineering, 200(17-20):1729-1745, 2011.

[7] Rodney Hill. Elastic properties of reinforced solids: some theoretical principles. Journal of the Mechanics and Physics of Solids, 11(5):357-372, 1963.

[8] J Mandel. Contribution théorique à l'étude de l'écrouissage et des lois de l'écoulement plastique. In Applied Mechanics, pages 502-509. Springer, 1966.

[9] M. Danielsson, D. M. Parks, and M. C. Boyce. Three-dimensional micromechanical modeling of voided polymeric materials. Journal of the Mechanics and Physics of Solids, 50(2):351-379, 2002. ISSN 00225096. doi: 10.1016/S0022-5096(01)00060-6.

[10] B. S. Kirk, J. W. Peterson, R. H. Stogner, and G. F. Carey. libMesh: A C++ Library for Parallel Adaptive Mesh Refinement/Coarsening Simulations. Engineering with Computers, 22(3-4):237-254, 2006. https://doi .org/10.1007/s00366-006-0049-3.

[11] Cody J. Permann, Derek R. Gaston, David Andrš, Robert W. Carlsen, Fande Kong, Alexander D. Lindsay, Jason M. Miller, John W. Peterson, Andrew E. Slaughter, Roy H. 
Stogner, and Richard C. Martineau. MOOSE: Enabling massively parallel multiphysics simulation. SoftwareX, 11:100430, 2020. ISSN 2352-7110. doi: https://doi.org/10. 1016/j.softx.2020.100430. URL http://www.sciencedirect.com/science/article/ pii/S2352711019302973.

[12] MC Messner, A Rovinelli, DM Parks, and T-L Sham. Evaluation of statistical variation of microstructural properties and temperature effects on creep fracture of grade 91. Technical Report ANL-ART-143, Argonne National Lab.(ANL), Argonne, IL (United States), Lemont, IL, USA, 2018.

[13] A. Rovinelli, M. C. Messner, Guosheng Ye, and T.-L. Sham. Initial study of notch sensitivity of grade 91 using mechanisms motivated crystal plasticity finite element method. Technical Report ANL-ART-171, Argonne National Laboratory, Lemont, IL, USA, 2020.

[14] Kazuhiro Kimura, Kota Sawada, and Hideaki Kushima. Creep rupture ductility of creep strength enhanced ferritic steels. Journal of Pressure Vessel Technology, 134(3), 2012.

[15] Vinh Phu Nguyen. An open source program to generate zero-thickness cohesive interface elements. Advances in Engineering Software, 74:27-39, 2014.

[16] Vinh Phu Nguyen. Discontinuous galerkin/extrinsic cohesive zone modeling: Implementation caveats and applications in computational fracture mechanics. Engineering Fracture Mechanics, 128:37-68, 2014.

[17] Yi Chao Chen and Lewis Wheeler. Derivatives of the stretch and rotation tensors. Journal of Elasticity, 32(3):175-182, 1993. ISSN 03743535. doi: 10.1007/BF00131659.

[18] Argonne National Laboratory. NEML: the Nuclear Engineering material Model Library. https://github.com/Argonne-National-Laboratory/neml, 2019.

[19] T-L Sham and A Needleman. Effects of triaxial stressing on creep cavitation of grain boundaries. Acta Metallurgica, 31(6):919-926, jun 1983. ISSN 00016160. doi: 10.1016/0001-6160(83)90120-7. URL https://linkinghub.elsevier.com/retrieve/ pii/0001616083901207.

[20] Erik Van Der Giessen, M W D der Burg, A Needleman, and V Tvergaard. Void growth due to creep and grain boundary diffusion at high triaxialities. Journal of the Mechanics and Physics of Solids, 43(1):123-165, 1995. ISSN 00225096. doi: 10.1016/0022-5096(94) 00059-E.

[21] A. Rovinelli, M. C. Messner, and T.-L. Sham. Initial microstructural model for creepfatigue damage in grade 91 steel. Technical Report ANL-ART-202, Argonne National Laboratory, Lemont, IL, USA, 2020.

[22] Mark Christian Messner, Omar Nassif, Ran Ma, Timothy J Truster, Kristine Cochran, David Parks, and TL Sham. Combined crystal plasticity and grain boundary modeling of creep in ferritic-martensitic steels: Ii. the effect of stress and temperature on engineering 
and microstructural properties. Modelling and Simulation in Materials Science and Engineering, 27(7):075010, 2019.

[23] M. C. Messner, T. J. Truster, K. B. Cochran, D. M. Parks, and T.-L. Sham. FY17 status report on the micromechanical finite element modeling of creep fracture of Grade 91 steel. Technical Report ANL-ART-95, Argonne National Laboratory, 2017.

[24] R. J. Asaro. Crystal plasticity. Transactions of the ASME. Journal of Applied Mechanics, 50(4B):921-934, 1983.

[25] Kouichi Maruyama, Kota Sawada, and Jun ichi Koike. Strengthening mechanisms of creep resistant tempered martensitic steel. ISIJ International, 41(6):641-653, 2001. doi: 10.2355/isijinternational.41.641.

[26] Ritwik Bandyopadhyay, Veerappan Prithivirajan, and Michael D Sangid. Uncertainty quantification in the mechanical response of crystal plasticity simulations. JOM, 71(8): 2612-2624, 2019.

[27] Luboš Kloc and Václav Sklenička. Transition from power-law to viscous creep behaviour of p-91 type heat-resistant steel. Materials Science and Engineering: A, 234:962-965, 1997.

[28] RC Gifkins and KU Snowden. Mechanism for "viscous" grain-boundary sliding. Nature, 212(5065):916-917, 1966.

[29] E. S. McGarrity, P. M. Duxbury, and E. A. Holm. Statistical physics of grain-boundary engineering. Phys. Rev. E, 71:026102, Feb 2005. doi: 10.1103/PhysRevE.71.026102. URL https://link.aps.org/doi/10.1103/PhysRevE.71.026102.

[30] Fuhua Cao, Yong Jiang, Tao Hu, and Dengfeng Yin. Correlation of grain boundary extra free volume with vacancy and solute segregation at grain boundaries: a case study for $\mathrm{Al}$. Philosophical Magazine, 98(6):464-483, 2018. ISSN 14786443. doi: 10.1080/14786435. 2017.1408968. URL https://doi.org/10.1080/14786435.2017.1408968.

[31] Sutatch Ratanaphan, David L. Olmsted, Vasily V. Bulatov, Elizabeth A. Holm, Anthony D. Rollett, and Gregory S. Rohrer. Grain boundary energies in body-centered cubic metals. Acta Materialia, 88:346-354, 2015. ISSN 13596454. doi: 10.1016/j. actamat.2015.01.069. URL http://dx.doi.org/10.1016/j. actamat.2015.01.069.

[32] Meimei Li, K. Natesan, and Weiying Chen. Report on understanding and predicting effects of thermal aging on microstructure and tensile properties of grade 91 steel for structural components. Technical Report ANL-ART-108, Argonne National Laboratory, 82017.

[33] MA Linne, A Venkataraman, MD Sangid, and S Daly. Grain boundary sliding and slip transmission in high purity aluminum. Experimental Mechanics, 59(5):643-658, 2019. 
[34] Ajey Venkataraman, Marissa Linne, Samantha Daly, and Michael D Sangid. Criteria for the prevalence of grain boundary sliding as a deformation mechanism. Materialia, 8:100499, 2019.

[35] Vasily V Bulatov, Bryan W Reed, and Mukul Kumar. Grain boundary energy function for fcc metals. Acta Materialia, 65:161-175, 2014. 



\section{Argonne}

\section{Applied Materials Division}

Argonne National Laboratory

9700 South Cass Avenue, Bldg. 212

Argonne, IL 60439

www.anl.gov 